\title{
GENERALIZED RADIX REPRESENTATIONS AND DYNAMICAL SYSTEMS
}

\author{
SHIGEKI AKIYAMA, TIBOR BORBÉLY, HORST BRUNOTTE, ATTILA PETHŐ, \\ AND JÖRG M. THUSWALDNER
}

\begin{abstract}
In this paper we are concerned with families of dynamical systems which are related to generalized radix representations. The properties of these dynamical systems lead to new results on the characterization of bases of Pisot Number Systems as well as Canonical Number Systems.
\end{abstract}

\section{INTRODUCTION}

The present paper is devoted to the study of a certain dynamics on the set of integer vectors $\mathbb{Z}^{d}$, which is intimately related to generalized radix representations. In particular, this dynamics on $\mathbb{Z}^{d}$ will be useful for characterizing base numbers with certain finiteness properties. Furthermore, it reveals unexpected relations between different notions of radix representations. Although we will make no use of the theory of dynamical systems, the reader will find that this simple dynamical system on $\mathbb{Z}^{d}$ plays an essential role in understanding the global structure of various numeration systems.

The first kind of radix representations we are dealing with are the so-called $\beta$-expansions which go back to Rényi [34] and Parry [29]. Let $\beta>1$ be a non-integral real number and let $\mathcal{A}=$ $\{0,1, \ldots,\lfloor\beta\rfloor\}$ be the set of digits. Then each $\gamma \in[0, \infty)$ can be represented uniquely by

$$
\gamma=a_{m} \beta^{m}+a_{m-1} \beta^{m-1}+\cdots
$$

with $a_{i} \in \mathcal{A}$ such that

$$
0 \leq \gamma-\sum_{i=n}^{m} a_{i} \beta^{i}<\beta^{n}
$$

holds for all $n \leq m$. Since by condition (1.2) the digits $a_{i}$ are selected as large as possible, the representation in (1.1) is called the greedy expansion of $\gamma$ with respect to $\beta$.

For $\gamma \in[0,1)$ this greedy expansion can be given by the $\beta$-transformation

$$
T_{\beta}(\gamma)=\beta \gamma-\lfloor\beta \gamma\rfloor
$$

(cf. Rényi [34]). For 1 the $\beta$-transformation produces a different representation as the greedy algorithm. This representation is often called $d(1, \beta)$.

Parry [29] was the first to prove results on the set of admissible digit strings in these expansions. Schmidt [38] observed that in order to get ultimately periodic expansions for all $\gamma \in \mathbb{Q} \cap(0,1)$ it is necessary for $\beta$ to be a Pisot or a Salem number. On the other hand, Bertrand [9] and Schmidt [38] proved that if $\beta$ is a Pisot number then each expansion of $\gamma \in \mathbb{Q}(\beta) \cap[0, \infty)$ is ultimately periodic.

It is interesting to ask whether there exist base numbers $\beta$ which give rise to finite $\beta$-expansions for large classes of numbers. This problem was first studied in a systematical way by Frougny and

Date: June 11, 2008.

2000 Mathematics Subject Classification. 11A63.

Key words and phrases. beta expansion, canonical number system, periodic point, contracting polynomial, Pisot number.

The first author was supported by the Japanese Ministry of Education, Culture, Sports, Science and Technology, Grant-in Aid for fundamental research 14540015, 2002-2005.

The fourth author was supported partially by the Hungarian National Foundation for Scientific Research Grant Nos. T42985 and T38225, as well as HAS-JSPS bilateral project RC20318007.

The fifth author was supported by project S8310 of the Austrian Science Foundation. 
Solomyak [12]. Let $\operatorname{Fin}(\beta)$ be the set of positive real numbers having finite greedy expansion with respect to $\beta$. Following [12], we say that $\beta>1$ has property $(\mathrm{F})$ if

$$
\operatorname{Fin}(\beta)=\mathbb{Z}[1 / \beta] \cap[0, \infty),
$$

i.e. $\beta$ has property $(\mathrm{F})$ if all reasonable candidates have finite $\beta$-expansion. It is shown in $[12$, Lemma 1] that (F) can hold only for Pisot numbers $\beta$. On the other hand, not all Pisot numbers have property (F). A geometrical meaning of property (F) turned out to be of interest. Thurston [41] introduced the concept of dual tiling associated to greedy expansions, and the first author showed that the origin is an "exclusive" inner point of the central dual tile if and only if property (F) holds (see $[1,3,8]$ ). Making use of the inner point property Praggastis [33] constructed a natural extension of the $\beta$-transformation which gives a concrete Markoff partition of the associated symbolic dynamics.

Thus we are left with the problem of characterizing all Pisot numbers with property $(\mathrm{F})$. It turns out that this is a very difficult problem. There exist many partial results in the literature. For the case of quadratic Pisot numbers a complete characterization was given by Frougny and Solomyak [12]. But even the characterization of cubic Pisot numbers with property (F) is still unknown. Akiyama [2] could settle the case of cubic Pisot units. Furthermore, there are results on Pisot numbers of arbitrary degree. Let

$$
X^{d}-a_{1} X^{d-1}-\cdots-a_{d-1} X-a_{d}
$$

be the minimal polynomial of a Pisot number $\beta$. In [12] it is shown that if $\beta$ is a Pisot number with minimal polynomial (1.3) satisfying $a_{1} \geq \ldots \geq a_{d} \geq 1$ then $\beta$ fulfills property (F). Hollander [15] proved characterization results for $(\mathrm{F})$ under a condition on the representation $d(1, \beta)$. Namely, he could show that $\beta$ has property (F) if $d(1, \beta)=. d_{1} \ldots d_{l}$ with $d_{1}>d_{2}+\cdots+d_{l}$ (cf. [15, Theorem 3.4.2]).

One aim of the present paper is to give new ideas and results on the characterization of Pisot numbers with property $(\mathrm{F})$. However, we are not restricted to the notion of $\beta$-expansions. It turns out that our methods will also work for a different notion of radix representations, the so called canonical number systems.

An example of a canonical number system was first studied by Knuth [20, 21] and Penney [30]. In particular, they observed that the number $b=-1+\sqrt{-1}$ can be used as a base for a number system which admits finite representations for each Gaussian integer. This means that each nonzero $\gamma \in \mathbb{Z}[\sqrt{-1}]$ has a unique representation of the shape

$$
\gamma=c_{0}+c_{1} b+\cdots+c_{h} b^{h}
$$

with $c_{i} \in\{0,1\}(0 \leq i<h), c_{h}=1$ and $h \in \mathbb{N}$. This observation has been generalized and studied extensively in the last decades. Gilbert, Kátai, Kovács and Szabó (cf. [13, 16, 17, 18]) extended Knuth's notion to arbitrary quadratic number fields. For each quadratic number field $K$ with maximal order $\mathbb{Z}_{K}$ they could characterize all the elements $b \in \mathbb{Z}_{K}$ which can serve as a base of a number system in $\mathbb{Z}_{K}$ : If $\alpha$ be an algebraic integer then $b$ is called a base for $\mathbb{Z}[\alpha]$ if each non-zero $\gamma \in \mathbb{Z}[\alpha]$ admits a unique representation of the shape

$$
\gamma=c_{0}+c_{1} b+\cdots+c_{h} b^{h}
$$

with $c_{i} \in\{0,1, \ldots,|N(b)|-1\}$ and $c_{h} \neq 0(N(\cdot)$ is the norm of $b$ over $\mathbb{Q})$. Later, Kovács and Pethő [26] (see also Kovács [24]) extended this notion to arbitrary number fields and gave some partial results on the characterization of the bases. Their framework also includes the special case of the number field $\mathbb{Q}$, which has been studied by Grünwald [14]. Grünwald showed that an integer $b$ is a base of a number system in the number field $\mathbb{Q}$ if and only if $b \leq-2$.

The fourth author [31] observed that the above mentioned number systems can be defined without using number fields. It is this slightly more general notion which we will use in the present paper. We will recall the exact definition. Let

$$
P(X)=X^{d}+p_{d-1} X^{d-1}+\cdots+p_{1} X+p_{0} \in \mathbb{Z}[X], \quad \mathcal{N}=\left\{0,1, \ldots,\left|p_{0}\right|-1\right\}
$$


and $R:=\mathbb{Z}[X] / P(X) \mathbb{Z}[X]$ and denote the image of $X$ under the canonical epimorphism from $\mathbb{Z}[X]$ to $R$ by $x$. If every non-zero element $A(x) \in R$ can be written uniquely in the form

$$
A(x)=a_{0}+a_{1} x+\cdots+a_{\ell} x^{\ell}
$$

with $a_{0}, \ldots, a_{\ell} \in \mathcal{N}, a_{\ell} \neq 0$, we call $(P, \mathcal{N})$ a canonical number system (CNS for short). $P(X)$ is called base of the CNS $(P, \mathcal{N})$ or $C N S$ polynomial. To $\mathcal{N}$ we refer as the set of digits.

If $P(X)$ is a CNS polynomial each coset $A+P(X) \mathbb{Z}[X] \quad(A \in \mathbb{Z}[X])$ contains a polynomial with coefficients belonging to $\mathcal{N}$. The polynomial (1.4) will be called the CNS representation of this coset.

It is easy to see that for irreducible polynomials this notion of canonical number systems coincides with the one defined in algebraic number fields.

In this setting it is a fundamental problem to characterize all CNS polynomials. Slightly generalizing the above mentioned results by Gilbert, Kátai, Kovács and Szabó, Brunotte [11] gave a characterization of all quadratic CNS polynomials. For irreducible CNS polynomials of general degree, Kovács [24] proved that a polynomial

$$
X^{d}+p_{d-1} X^{d-1}+\cdots+p_{1} X+p_{0}
$$

is CNS if $p_{0} \geq 2$ and $p_{0} \geq p_{1} \geq \cdots \geq p_{d-1}>0$. More recently, Akiyama and Pethő [6], Scheicher and Thuswaldner [36] as well as Akiyama and Rao [7] showed characterization results under the condition $p_{0}>\left|p_{1}\right|+\cdots+\left|p_{d-1}\right|$. These results are analogous to the above mentioned theorems of Frougny and Solomyak [12] as well as Hollander [15].

Various variants of canonical number systems have been studied in the literature. Kovács [23] studied number systems with integer digits in rings. Pethő [32] considered simultaneous representation of several elements. Scheicher and Thuswaldner [37] investigated number systems in polynomial rings over finite fields (cf. also [26]). In the finite field setting things turn out to be easier and complete results on the structure of the number systems are tractable.

Variations of the set of digits for a given CNS polynomial have been considered by Kovács and Pethő [25] and later by Michalek [28]. Kátai [19] characterized digit sets for imaginary quadratic integers.

The second aim of this paper is to develop methods for pushing forward the characterization of CNS polynomials.

We have stated two different characterization problems. It turns out that they are strongly related to each other and can be unified by considering the following dynamical system. Let $d \geq 1$ be an integer and $\mathbf{r}=\left(r_{1}, \ldots, r_{d}\right) \in \mathbb{R}^{d}$. To $\mathbf{r}$ we associate the mapping $\tau_{\mathbf{r}}: \mathbb{Z}^{d} \rightarrow \mathbb{Z}^{d}$ in the following way: if $\mathbf{a}=\left(a_{1}, \ldots, a_{d}\right) \in \mathbb{Z}^{d}$ then let

$$
\tau_{\mathbf{r}}(\mathbf{a})=\left(a_{2}, \ldots, a_{d},-\lfloor\mathbf{r a}\rfloor\right),
$$

where $\mathbf{r a}=r_{1} a_{1}+\cdots+r_{d} a_{d}$, i.e. the inner product of the vectors $\mathbf{r}$ and $\mathbf{a}$.

Let $\mathbf{r}$ be fixed. It will turn out that (under certain conditions) $\mathbf{r}$ gives rise to an algebraic number $\beta$ with property $(\mathrm{F})$ as well as to a CNS polynomial $P(X)$ if and only if

$$
\text { for all } \mathbf{a} \in \mathbb{Z}^{d} \text { there exists } k>0 \text { with } \tau_{\mathbf{r}}^{k}(\mathbf{a})=0 \text {. }
$$

If (1.5) holds, we will call $\tau_{\mathbf{r}}$ a shift radix system ( $S R S$ for short). For simplicity, we write $0=(0, \ldots, 0)$.

The details of this correspondence will be given in the next sections. In Section 2 we explicitely state the relation between SRS and $\beta$-expansions and introduce two subsets of the real $d$-dimensional space which will play a fundamental role in the sequel. In Section 3 we deal with the relation between SRS and CNS and motivate our discussion by some remarks on the historical development of these concepts. In Section 4 basic properties of SRS are explained which in Section 5 lead to an efficient algorithm for the determination of SRS; our main tools here come from geometry. Geometrical concepts are further exploited in the last two parts of the paper where we study periodic properties (Section 6) and so-called "critical points" (Section 7). These geometrical considerations shed some light on the difficulties of a complete description of SRS. 


\section{The ReLation Between Shift RadiX Systems and $\beta$-eXPansions}

Before establishing the correspondence between SRS and $\beta$-expansions we have to define two sets related to the behavior of the periods of $\tau_{\mathbf{r}}$. Let

$$
\begin{aligned}
& \mathcal{D}_{d}^{0}:=\left\{\mathbf{r} \in \mathbb{R}^{d} \mid \forall \mathbf{a} \in \mathbb{Z}^{d} \exists k>0: \tau_{\mathbf{r}}^{k}(\mathbf{a})=0\right\} \text { and } \\
& \mathcal{D}_{d}:=\left\{\mathbf{r} \in \mathbb{R}^{d} \mid \forall \mathbf{a} \in \mathbb{Z}^{d} \text { the sequence }\left\{\tau_{\mathbf{r}}^{k}(\mathbf{a})\right\}_{k \geq 0} \text { is ultimately periodic }\right\} .
\end{aligned}
$$

The next theorem is basically due to Hollander [15] except the notion of SRS. For the convenience of the reader, we give its proof.

Theorem 2.1. Let $\beta>1$ be an algebraic integer with minimal polynomial $X^{d}-a_{1} X^{d-1}-\cdots-$ $a_{d-1} X-a_{d}$. Set

$$
\begin{aligned}
& r_{1}:=1, \\
& r_{j}:=a_{j} \beta^{-1}+a_{j+1} \beta^{-2}+\cdots+a_{d} \beta^{j-d-1} \quad(2 \leq j \leq d) .
\end{aligned}
$$

Then $\beta$ has property $(F)$ if and only if $\left(r_{d}, \ldots, r_{2}\right) \in \mathcal{D}_{d-1}^{0}$.

Proof. We claim that $\beta$ admits property (F) if and only if every element of $\mathbb{Z}[\beta] \cap[0, \infty)$ has finite $\beta$-expansion. Indeed, if $\gamma \in \mathbb{Z}[1 / \beta] \cap[0, \infty)$ then there exists an integer $k$ such that $\gamma^{\prime}:=\gamma \beta^{k} \in$ $\mathbb{Z}[\beta] \cap[0, \infty)$. The claim now follows from the fact that the $\beta$-expansions of $\gamma$ and $\gamma^{\prime}$ have the same digit string.

Thus we have to deal only with elements of the ring $\mathbb{Z}[\beta]$. This ring is generated by $\left\{1, \beta, \ldots, \beta^{d-1}\right\}$ as a $\mathbb{Z}$ - module. The same is true for $\left\{r_{1}, \ldots, r_{d}\right\}$. Thus each element $\gamma \in \mathbb{Z}[\beta]$ can be expressed in the form $\gamma=\sum_{i=1}^{d} z_{d-i} r_{i}$, where $z_{0}, \ldots, z_{d-1} \in \mathbb{Z}$. Assume w.l.o.g. that $\gamma \in \mathbb{Z}[\beta] \cap[0,1)$. Then the $\beta$-transform of $\gamma$ can be written as $T_{\beta}(\gamma)=\sum_{i=1}^{d} z_{d+1-i} r_{i}$ with $z_{d}$ satisfying

$$
0 \leq z_{d} r_{1}+z_{d-1} r_{2}+\cdots+z_{1} r_{d}<1
$$

and $z_{d}$ is uniquely determined by this inequality. Let $\mathbf{r}:=\left(r_{d}, \ldots, r_{2}\right)$, then $z_{d}=-\left\lfloor z_{d-1} r_{2}+\cdots+\right.$ $\left.z_{1} r_{d}\right\rfloor$. By the definition of $\tau_{\mathbf{r}}$ we see that

$$
\tau_{\mathbf{r}}\left(z_{1}, \ldots, z_{d-1}\right)=\left(z_{2}, \ldots, z_{d}\right) .
$$

The relation between $\beta$-expansions with respect to a Pisot number $\beta$ and SRS is more closely understood by the next formula

$$
X^{d}-a_{1} X^{d-1}-a_{2} X^{d-2}-\cdots-a_{d}=(X-\beta)\left(X^{d-1}+r_{2} X^{d-2}+r_{3} X^{d-3}+\cdots+r_{d}\right) .
$$

The polynomial $X^{d-1}+r_{2} X^{d-2}+r_{3} X^{d-3}+\cdots+r_{d}$ is contractive, i.e., all roots of it are less than one in modulus. By using this fact, we will see in Lemma 4.2 that the SRS algorithm associated to $\left(r_{d}, \ldots, r_{2}\right)$ always yields eventually periodic orbits. This means that the $\beta$-expansion of each positive element of $\mathbb{Z}[\beta]$ must be eventually periodic. This assertion is easily proven even in $\mathbb{Q}(\beta)$ in a similar manner which reproves the result of [9] and [38] stated in the introduction.

To illustrate the difficulty of the characterization problem of cubic Pisot numbers with property (F) we refer the reader to Figure 1. By using Lemma 4.1, we can show

$$
\mathcal{D}_{2} \subset\{(u, v)|u \geq| v \mid-1, u \leq 1\} .
$$

Moreover $\mathcal{D}_{2}^{0}$ is a subset of the region

$$
\mathcal{D}_{2}^{\prime}=\{(u, v) \mid 0 \leq u \leq 1, u \geq-v, u \geq v-1\} .
$$

(See Example 4.7.)

In Figure 1 we present $\mathcal{D}_{2}^{\prime}$. As an approximation of $\mathcal{D}_{2}^{0}$ we show all $(u, v)=\left(\frac{p_{1}}{174}, \frac{p_{2}}{174}\right) \in \mathcal{D}_{2}^{\prime}$ with $p_{1}, p_{2} \in \mathbb{Z}$. The gray points belong to $\mathcal{D}_{2}^{0}$ while the black points belong to $\mathcal{D}_{2}^{\prime} \backslash \mathcal{D}_{2}^{0}$. The picture is presented in the coordinate system $(v, u)$ to be easier comparable to Figure 2 in Section 3. 


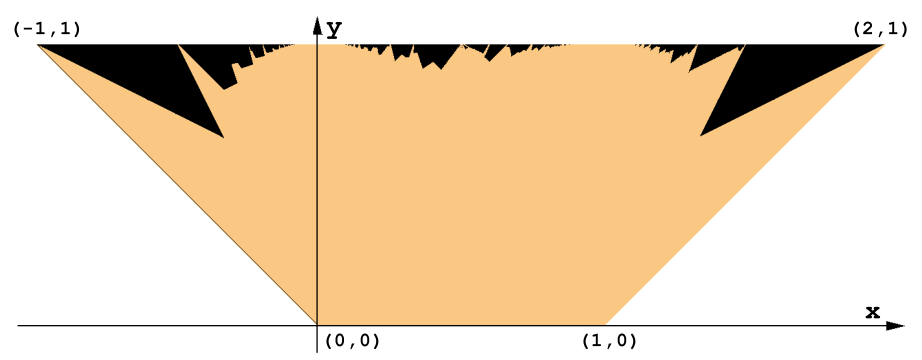

FiguRE 1. An approximation of $\mathcal{D}_{2}^{0}$

\section{THE RELATION BETWEEN SHIFT RADIX SYSTEMS AND CANONICAL NUMBER SYSTEMS}

In this section we deal with the relations between SRS and CNS. We start with some easy remarks on a special case in order to show what is going on in general.

Let $q \geq 2$ be an integer. Then each $n \in \mathbb{N}$ has a representation of the shape

$$
n=c_{0}+c_{1} q+\cdots+c_{h} q^{h}
$$

with $h \in \mathbb{N}$ and $0 \leq c_{j}<q(0 \leq j \leq h)$. This can be modelled by means of the following dynamical system. Set

$$
T_{q}(n):=\frac{n-d}{q}
$$

where $0 \leq d<q$ has the property that $n \equiv d(\bmod q)$. Then the fact that each positive integer $n$ has a representation of the shape (3.1) can be expressed by saying that

$$
\text { for all } n \in \mathbb{N} \text { there exists an } h \in \mathbb{N} \text { such that } T_{q}^{h}(n)=0 \text {. }
$$

For the convenience of the reader we recapitulate several notations from the introduction. Let $P(X)=p_{d} X^{d}+\cdots+p_{0} \in \mathbb{Z}[X]$ with $p_{0} \geq 2$ and $p_{d}=1$, and set $\mathcal{N}=\left\{0,1, \ldots, p_{0}-\right.$ $1\}$. Furthermore, denote the image of $X$ under the canonical epimorphism from $\mathbb{Z}[X]$ to $R:=$ $\mathbb{Z}[X] / P(X) \mathbb{Z}[X]$ by $x$. Since $p_{d}=1$ it is clear that each coset of $R$ has a unique element of degree at most $d-1$, say

$$
A(x)=A_{0}+A_{1} x+\cdots+A_{d-1} x^{d-1} .
$$

To transform $A(x)$ into a CNS representation it is straightforward to use the following backward division algorithm. Let $\mathbb{Z}^{\prime}[X]=\{A(X) \in \mathbb{Z}[X]$ : $\operatorname{deg} A<d\}$ and

$$
T_{P}(A)=\sum_{i=0}^{d-1}\left(A_{i+1}-q p_{i+1}\right) X^{i},
$$

where $A_{d}=0$ and $q=\left\lfloor A_{0} / p_{0}\right\rfloor$. Then $T_{P}: \mathbb{Z}^{\prime}[X] \rightarrow \mathbb{Z}^{\prime}[X]$ and

$$
A(x)=\left(A_{0}-q p_{0}\right)+x T_{P}(A) \text {, where } A_{0}-q p_{0} \in \mathcal{N} \text {. }
$$

This backward division process can become divergent (e.g. $A(X)=-1$ for $P(X)=X^{2}+4 X+2$ ) or ultimately periodic (e.g. $A(X)=-1$ for $P(X)=X^{2}-2 X+2$ ) or can terminate after finitely many steps (e.g. $A(X)=-1$ for $P(X)=X^{2}+2 X+2$ ).

Let

$$
\Pi(P)=\left\{A: T_{P}^{\ell}(A)=A \text { for some } \ell>0\right\}
$$

denote the set of periodic points of the mapping $T_{P}$. It is clear that we always have $0 \in \Pi(P)$. We define

$$
\begin{aligned}
\mathcal{C}_{d}^{0} & :=\left\{\left(p_{0}, p_{1}, \ldots, p_{d-1}\right) \in \mathbb{Z}^{d} \mid \forall A \in \mathbb{Z}^{\prime}[X] \quad \exists \ell \in \mathbb{N} \quad T_{X^{d}+p_{d-1} X^{d-1}+\cdots+p_{0}}^{\ell}(A)=0\right\} \\
\text { and } & \\
\mathcal{C}_{d} & :=\left\{\left(p_{0}, p_{1}, \ldots, p_{d-1}\right) \in \mathbb{Z}^{d} \mid T_{X^{d}+p_{d-1} X^{d-1}+\cdots+p_{0}} \text { has only finite orbits }\right\} .
\end{aligned}
$$

Clearly, we have $\mathcal{C}_{d}^{0} \subset \mathcal{C}_{d}$. 
The mapping $T_{P}$ is intimately related to canonical number systems. In accordance with the easy example at the beginning of the present section, a polynomial is a CNS polynomial if and only if $T_{P}$ has only finite orbits and $\Pi(P)=\{0\}$. Furthermore, to obtain the CNS representation of a coset $A(x)$ one has to compute the iterates $A, T_{P}(A), T_{P}^{2}(A), \ldots$ until $T_{P}^{\ell}(A)=0$ for some $\ell>0$. Hence, it suffices to study the map $T_{P}$ in order to decide whether $P(X)$ is a CNS polynomial or not.

A fundamental problem consists in describing the set of all bases of CNS. This is tantamount to describing the sets $\mathcal{C}_{d}^{0}$. By Gilbert, Kátai, Kovács and Szabó (cf. [13, 16, 17, 18]) it was shown that

$$
\mathcal{C}_{2}^{0}=\left\{\left(p_{0}, p_{1}\right) \mid-1 \leq p_{1} \leq p_{0} \geq 2\right\}
$$

For $d \geq 3$ this problem seems to be very difficult. Even the set $\mathcal{C}_{3}^{0}$ has not been characterized up to now (cf. Akiyama et al. [4] for partial results).

It is convenient to replace $T_{P}$ by the conjugate mapping $\tilde{T}_{P}: \mathbb{Z}^{d} \rightarrow \mathbb{Z}^{d}$ defined as

$$
\tilde{T}_{P}\left(\left(A_{0}, \ldots, A_{d-1}\right)\right)=\left(A_{1}-q p_{1}, \ldots, A_{d-1}-q p_{d-1},-q p_{d}\right), q=\left\lfloor A_{0} / p_{0}\right\rfloor .
$$

Brunotte [11] (and independently Scheicher and Thuswaldner [36]) observed that the basis transformation

$$
\begin{aligned}
\left\{1, x, \ldots, x^{d-1}\right\} & \rightarrow\left\{w_{1}, \ldots, w_{d}\right\} \\
w_{j} & =\sum_{i=d-j+1}^{d} p_{i} x^{i+j-d-1} \quad(j \in\{1, \ldots, d\})
\end{aligned}
$$

of $R$ implies a nicer and much better applicable transformation than $\tilde{T}_{P}$ is. Indeed, if

$$
\begin{aligned}
A(x) & =\sum_{j=1}^{d} a_{j} w_{j}, \text { then } \\
\tilde{T}_{P}(A) & =-t w_{d}+\sum_{j=1}^{d-1} a_{j+1} w_{j}, \text { where } t=\left\lfloor\frac{p_{1} a_{d}+\cdots+p_{d} a_{1}}{p_{0}}\right\rfloor .
\end{aligned}
$$

Hence, $\tilde{T}_{P}$ implies the mapping $\tau_{P}: \mathbb{Z}^{d} \rightarrow \mathbb{Z}^{d}$

$$
\tau_{P}(\mathbf{A})=\left(a_{2}, \ldots, a_{d},-\left\lfloor\frac{p_{1} a_{d}+\cdots+p_{d} a_{1}}{p_{0}}\right\rfloor\right)
$$

where $\mathbf{A}=\left(a_{1}, \ldots, a_{d}\right)$. The mapping $\tau_{P}$ will be called Brunotte's mapping.

Theorem 3.1. Let $P(X):=X^{d}+p_{d-1} X^{d-1}+\cdots+p_{1} X+p_{0} \in \mathbb{Z}[X]$. Then $\left(p_{0}, \ldots, p_{d-1}\right) \in \mathcal{C}_{d}^{0}$ if and only if $\mathbf{r}:=\left(\frac{1}{p_{0}}, \frac{p_{d-1}}{p_{0}}, \ldots, \frac{p_{1}}{p_{0}}\right) \in \mathcal{D}_{d}^{0}$.

Proof. According to the above considerations $P(X)$ is a CNS polynomial if and only if for all $\mathbf{A}:=\left(a_{1}, \ldots, a_{d}\right) \in \mathbb{Z}^{d}$ there exists a $k>0$ such that $\tau_{P}^{k}(\mathbf{A})=0$. Using the definitions of $\tau_{P}$ and $\tau_{\mathbf{r}}$ and noting that $p_{d}=1$ we get

$$
\begin{aligned}
\tau_{P}(\mathbf{A}) & =\left(a_{2}, \ldots, a_{d},-\left\lfloor\frac{p_{1} a_{d}+\cdots+p_{d} a_{1}}{p_{0}}\right\rfloor\right) \\
& =\left(a_{2}, \ldots, a_{d},-\left\lfloor\frac{p_{d}}{p_{0}} a_{1}+\cdots+\frac{p_{1}}{p_{0}} a_{d}\right\rfloor\right) \\
& =\tau_{\mathbf{r}}(\mathbf{A}) .
\end{aligned}
$$

Thus $P(X)$ is a CNS polynomial if and only if for all $\mathbf{A} \in \mathbb{Z}^{d}$ there exists a $k>0$ such that $\tau_{\mathbf{r}}^{k}(\mathbf{A})=0$. By the definition of $\tau_{\mathbf{r}}$ this is just the claimed result.

This theorem gives an important relation between the sets $\mathcal{C}_{d}^{0}$ and $\mathcal{D}_{d}^{0}$. It is easy to see from the definition of $\tau_{\mathbf{r}}$ that

$$
\mathcal{D}_{d-1}^{0}=\left\{\left(r_{1}, \ldots, r_{d}\right) \in \mathcal{D}_{d}^{0} \mid r_{1}=0\right\}
$$




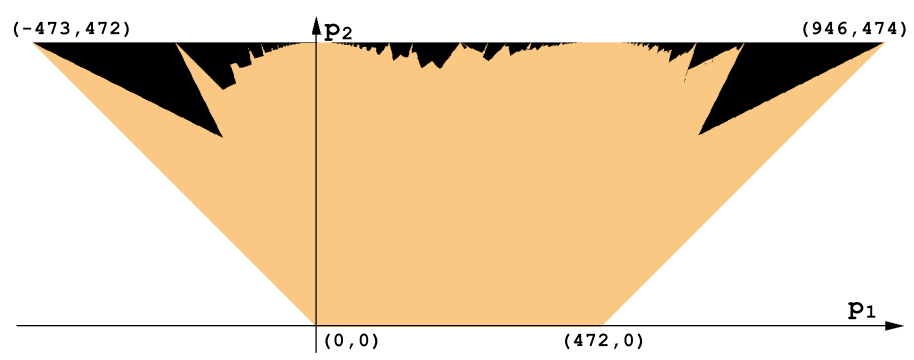

FiguRE 2. An approximation of $\mathcal{C}_{3}^{0}$ with $p_{0}=474$

For large $p_{0}$ the vectors $\mathbf{r}$ in the previous theorem have rather small first coordinate. In view of the last equation this means that these points are "near" to $\mathcal{D}_{d-1}^{0}$. Thus we may expect that a portion of the set $\mathcal{C}_{d}^{0}$ having large, fixed coordinate $p_{0}$ looks similar to $\mathcal{D}_{d-1}^{0}$.

Figure 2 shows a picture of the elements of $\mathcal{C}_{3}^{0}$ having $p_{0}=474$. The elements of $\mathcal{C}_{3}^{0}$ are indicated in grey. The black points correspond to a certain portion of the set $\mathcal{C}_{3} \backslash \mathcal{C}_{3}^{0}$ selected in a similar way as $\mathcal{D}_{2}^{\prime}$ in Figure 1. In fact one can see that this set is very similar to our approximation of $\mathcal{D}_{2}^{0}$.

\section{BASIC PROPERTIES OF SHIFT RADIX SYSTEMS}

Up to now we established the relation between two well known notions of radix representations and SRS. In what follows we want to study various properties of SRS. In view of the results of the previous sections these properties will carry over to $\beta$-expansions as well as to CNS.

For a matrix $M$ denote the spectral radius by $\rho(M)$, i.e., the maximum absolute value of eigenvalues of $M$. Take a real number $\tilde{\rho}>\rho(M)$. For a vector $\mathbf{v},\|\mathbf{v}\|$ shall fix a norm having the property $\|M \mathbf{v}\| \leq \tilde{\rho} \cdot\|\mathbf{v}\|$ for any $\mathbf{v} \in \mathbb{R}^{d}$ (e.g. see the formula (3.2) of [27]). Note that $\tilde{\rho}$ can be chosen arbitrarily close to $\rho(M)$.

For $\mathbf{r}=\left(r_{1}, \ldots, r_{d}\right) \in \mathcal{D}_{d}$ let

$$
R(\mathbf{r}):=\left(\begin{array}{ccccc}
0 & 1 & 0 & \cdots & 0 \\
\vdots & \ddots & \ddots & \ddots & \vdots \\
\vdots & & \ddots & \ddots & 0 \\
0 & \cdots & \cdots & 0 & 1 \\
-r_{1} & -r_{2} & \cdots & \cdots & -r_{d}
\end{array}\right)
$$

We start with a very easy condition on a vector $\mathbf{r} \in \mathbb{R}^{d}$ to be an SRS. The analogue for CNS is well known and can be found for instance in Gilbert [13].

Lemma 4.1. Let $d \in \mathbb{N}$. If $\mathbf{r}=\left(r_{1}, \ldots, r_{d}\right) \in \mathcal{D}_{d}$ then the spectral radius of $R(\mathbf{r})$ is less than or equal to 1.

Proof. Note that this statement was proved by Gilbert ([13], Proposition 3) for $R(\mathbf{r})$ diagonalizable. Assume that $\gamma$ is an eigenvalue of $R(\mathbf{r})$ with $|\gamma|>1$ and $\mathbf{u}$ is one of its "left" eigenvectors. Let $|\cdot|$ be the Euclidean norm. Then the $n$-th iterate $\tau_{\mathbf{r}}^{n}(\mathbf{x})$ of the integer vector $\mathbf{x}$ is

$$
\tau_{\mathbf{r}}^{n}(\mathbf{x})=R(\mathbf{r})^{n} \mathbf{x}+\sum_{i=1}^{n} R(\mathbf{r})^{n-i} \mathbf{v}_{i}
$$

with $\left|\mathbf{v}_{i}\right|<1$. There exists an absolute constant $k$ such that $\left|\mathbf{u v}_{i}\right| \leq k$ for any $\mathbf{v}_{i}$. Choose the integer vector $\mathbf{x}$ such that $|\mathbf{u x}|>(k+1) /(|\gamma|-1)$. Multiplying (4.2) by $\mathbf{u}$ from the left, we get

$$
\left|\mathbf{u} \tau_{\mathbf{r}}^{n}(\mathbf{x})\right|=\left|\gamma^{n} \mathbf{u x}+\sum_{i=1}^{n} \gamma^{n-i} \mathbf{u} \mathbf{v}_{i}\right|>c|\gamma|^{n}
$$

with a positive $c$. Thus the sequence $\tau_{\mathbf{r}}^{n}(\mathbf{x})$ is not eventually periodic. 
Because of this result we will emphasize especially on contracting polynomials. In the opposite direction we get the following result.

Lemma 4.2. Let $\mathbf{r} \in \mathbb{R}^{d}$ such that the spectral radius of $R(\mathbf{r})$ is less than 1 . Then

(1) $\mathbf{r} \in \mathcal{D}_{d}$ and

(2) if $\mathbf{a}$ is periodic for $\tau_{\mathbf{r}}$ and $\tilde{\rho}$ is less than 1 then $\|\mathbf{a}\| \leq \frac{1}{1-\tilde{\rho}}$.

Proof. By induction we have

$$
\left\|\tau_{\mathbf{r}}{ }^{k}(\mathbf{a})\right\| \leq \tilde{\rho}^{k}\|\mathbf{a}\|+\sum_{j=0}^{k-1} \tilde{\rho}^{j}
$$

for $k \in \mathbb{N}$ which implies

$$
\left\|\tau_{\mathbf{r}}{ }^{k}(\mathbf{a})\right\| \leq \tilde{\rho}^{k}\|\mathbf{a}\|+\frac{1}{1-\tilde{\rho}} .
$$

Thus the subset $\left\{\tau_{\mathbf{r}}{ }^{k}(\mathbf{a}): k \in \mathbb{N}\right\}$ of $\mathbb{Z}^{d}$ is bounded so that the above sequence must be ultimately periodic.

If there exists a positive $l \in \mathbb{N}$ with $\tau_{\mathbf{r}}^{l}(\mathbf{a})=\mathbf{a}$ then using (4.3) we see

$$
\|\mathbf{a}\|=\left\|\tau_{\mathbf{r}}^{k l}(\mathbf{a})\right\| \leq \tilde{\rho}^{k l}\|\mathbf{a}\|+\frac{1}{1-\tilde{\rho}}
$$

which implies $\|\mathbf{a}\| \leq \frac{1}{1-\tilde{\rho}}$.

Lemma 4.3. $\partial \mathcal{D}_{d}=\left\{\mathbf{r} \in \mathbb{R}^{d} \mid \rho(R(\mathbf{r}))=1\right\}$.

Proof. Let $\mathbf{r} \in \partial \mathcal{D}_{d}$ and assume $\rho(R(\mathbf{r}))<1$. The map $\mu$ which sends a real monic polynomial of degree $d$ to the maximal absolute value of its roots is continous. Hence, using Lemma 4.2 we can find a neighborhood of $\mathbf{r}$ lying in $\mathcal{D}_{d}$. Thus $\mathbf{r}$ belongs to the interior of $\mathcal{D}_{d}$ which contradicts our assumption. Let us suppose now that $\rho(R(\mathbf{r}))>1$ and that $\left(\mathbf{r}^{(k)}\right)_{k \in \mathbb{N}}$ is a sequence in $\mathcal{D}_{d}$ which converges to $\mathbf{r}$. By the continuity of $\mu$ there must be some $k \in \mathbb{N}$ such that $\rho\left(R\left(\mathbf{r}^{(k)}\right)\right)>1$ in contrast to Lemma 4.1 .

On the other hand let $\mathbf{r} \in \mathbb{R}^{d}$ with $\rho(R(\mathbf{r}))=1$. We need the following observation: If $\varepsilon>0$ and $f$ is a real monic polynomial with $\mu(f)=1$ then there exist real monic polynomials $g_{1}, g_{2}$ of the same degree as $f$ with $\mu\left(g_{1}\right)<1$ and $\mu\left(g_{2}\right)>1$ such that the absolute values of the differences of the corresponding coefficients of $f$ and $g_{1}$ as well as of $f$ and $g_{2}$ are less than $\varepsilon$.

Certainly, $\mathbf{r}$ is not an element of the interior of $\mathcal{D}_{d}$ because otherwise by this observation we could find some $\mathbf{s} \in \mathcal{D}_{d}$ with $\rho(R(\mathbf{s}))>1$ contradicting Lemma 4.1. Finally, we see $\mathbf{r} \in \overline{\mathcal{D}}_{d}$ by using Lemma 4.2 and the above observation.

We will now adopt the following notations. Let $\mathbf{r} \in \mathbb{R}^{d}$ and let $\mathbf{a}=\left(a_{1}, \ldots, a_{d}\right)$ be a non zero periodic point of period length $L$. Then we have $\mathbf{a}=\tau_{\mathbf{r}}^{L}(\mathbf{a})$. Suppose this period runs through the points

$$
\tau_{\mathbf{r}}^{j}(\mathbf{a})=\left(a_{1+j}, \ldots, a_{d+j}\right) \quad(0 \leq j \leq L-1),
$$

where $a_{1+L}=a_{1}, \ldots, a_{d+L}=a_{d}$ (note that the structure of the entries follows from the definition of $\tau_{\mathbf{r}}$ ). Then we will sometimes write this in the form

$$
\left(a_{1}, \ldots, a_{d}\right) \rightarrow\left(a_{2}, \ldots, a_{d+1}\right) \rightarrow \cdots \rightarrow\left(a_{1+L}, \ldots, a_{d+L}\right)=\left(a_{1}, \ldots, a_{d}\right) .
$$

Alternatively, the following shorter version will also be used:

$$
\left(a_{1}, \ldots, a_{d}\right) ; a_{d+1}, \ldots, a_{L} .
$$

If a period occurs for some $\tau_{\mathbf{r}}$ with $\mathbf{r} \in \mathbb{R}^{d}$, we will call it for short a period of $\mathcal{D}_{d}$. The same notations are used for periods of the mapping $\tau_{P}$. Moreover, a period $\pi$ of $\mathcal{C}_{d}$ is called a period for $p_{0}$ if there exists a polynomial $P(X) \in \mathbb{Z}[X]$ of degree $d$ with absolute coefficient $p_{0}$ such that $\tau_{P}$ has period $\pi$.

We now start the investigation of the sets $\mathcal{D}_{d}^{0}(d \geq 1)$. For $d=1$ things turn out to be easy. We can show the following result. 
Proposition 4.4. For $d=1$ we have

$$
\mathcal{D}_{1}^{0}=[0,1) .
$$

Proof. If $d=1$ then $\tau_{r}^{k}(a)$ can tend to 0 only if $|r| \leq 1 .-1<r<0$ is impossible because (1) is a period in this case. If $r=1$ then $\tau_{r}(1)=-1$ and, hence, $\tau_{r}^{2}(1)=1$. We conclude that $\mathcal{D}_{1}^{0}=[0,1)$, since for $0 \leq r<1$ the convergence to zero is obvious.

Remark 4.5. Combining Theorem 3.1 with Proposition 4.4 yields that $\mathcal{C}_{1}^{0}=\left\{p_{0} \mid p_{0} \geq 2\right\}$. This is Grünwald's result [14].

As mentioned before, already the case $d=2$ seems to be very hard as it is related to the set depicted in Figure 1.

The first general result shows a useful convexity property of $\tau_{\mathbf{r}}$.

Theorem 4.6. Let $\mathbf{r}_{1}, \ldots, \mathbf{r}_{k} \in \mathbb{R}^{d}$ and $\mathbf{a} \in \mathbb{Z}^{d}$ be such that $\tau_{\mathbf{r}_{1}}(\mathbf{a})=\cdots=\tau_{\mathbf{r}_{k}}(\mathbf{a})$. Let $\mathbf{s}$ be any convex linear combination of $\mathbf{r}_{1}, \ldots, \mathbf{r}_{k}$. Then we have $\tau_{\mathbf{s}}(\mathbf{a})=\tau_{\mathbf{r}_{1}}(\mathbf{a})=\cdots=\tau_{\mathbf{r}_{k}}(\mathbf{a})$.

Proof. By assumption we have $0 \leq \mathbf{r}_{i} \mathbf{a}-\left\lfloor\mathbf{r}_{1} \mathbf{a}\right\rfloor<1$ for $i=1, \ldots, k$. The set $K=\{\mathbf{x}=$ $\left.\left(x_{1}, \ldots, x_{d}\right) \in \mathbb{R}^{d} \mid 0 \leq \mathbf{x a}-\left\lfloor\mathbf{r}_{1} \mathbf{a}\right\rfloor<1\right\}$ is obviously convex. Thus for each convex linear combination $\mathbf{s}=\left(s_{1}, \ldots, s_{d}\right)$ of $\mathbf{r}_{1}, \ldots, \mathbf{r}_{k}$ we have $\mathbf{s} \in K$. This shows

$$
0 \leq a_{1} s_{1}+\cdots+a_{d} s_{d}-\left\lfloor\mathbf{r}_{1} \mathbf{a}\right\rfloor<1
$$

which implies $\tau_{\mathbf{s}}(\mathbf{a})=\tau_{\mathbf{r}_{1}}(\mathbf{a})$.

The same idea allows us to show that the set $\mathcal{D}_{d}^{0}$ can be constructed from the set $\mathcal{D}_{d}$ by "cutting out" families of convex polyhedra. In Section 7 we will see that these families have to be infinite for all $d \geq 2$.

Let $d \in \mathbb{N}$ and

$$
\left(a_{1+j}, \ldots, a_{d+j}\right) \quad(0 \leq j \leq L-1),
$$

with $a_{L+1}=a_{1}, \ldots, a_{L+d}=a_{d}$ be vectors of $\mathbb{Z}^{d}$. We ask for which $\mathbf{r}=\left(r_{1}, \ldots, r_{d}\right) \in \mathbb{R}^{d}$ these vectors form a period $\pi$ of $\mathcal{D}_{d}$. By the definition of $\tau_{\mathbf{r}}$ this is the case if and only if the inequalities

$$
0 \leq r_{1} a_{1+j}+\cdots+r_{d} a_{d+j}+a_{d+j+1}<1 \quad(0 \leq j \leq L-1)
$$

hold simultaneously. As each inequality gives an upper/lower half of hyperplanes in $\mathbb{R}^{d}$, it is easy to see that (4.5) defines a (possibly degenerated) convex polyhedron. We call this polyhedron $\mathcal{P}(\pi)$.

Since $\mathbf{r} \in \mathcal{D}_{d}^{0}$ if and only if $\tau_{\mathbf{r}}$ has 0 as its only period we conclude that

$$
\mathcal{D}_{d}^{0}=\mathcal{D}_{d} \backslash \bigcup_{\pi \neq 0} \mathcal{P}(\pi)
$$

where the union is extended over all families of vectors $\pi$ of the shape (4.4). We call the family of (non-empty) polyhedra corresponding to this choice the family of cutout polyhedra of $\mathcal{D}_{d}^{0}$.

Example 4.7. Let $\pi_{1}$ be $(1,1) \rightarrow(1,1)$ and $\pi_{2}$ be $(1,0) \rightarrow(0,1) \rightarrow(1,0)$. Then we have

$$
P\left(\pi_{1}\right)=\{(u, v) \mid-v-1 \leq u<-v\}, \quad P\left(\pi_{2}\right)=\{(u, v) \mid-1 \leq u<0,0 \leq v<1\}
$$

which makes plausible that $\mathcal{D}_{2}^{0} \subset \mathcal{D}_{2}^{\prime}$.

Example 4.8. Let $\pi$ be a period of length 5 in $\mathbb{Z}^{2}$ given by

$$
(-1,-1) \rightarrow(-1,1) \rightarrow(1,2) \rightarrow(2,1) \rightarrow(1,-1) \rightarrow(-1,-1)
$$

Then $P(\pi)$ is given by 10 inequalities. Summing up all together we have a triangle:

$$
P(\pi)=\left\{(u, v) \mid u \geq \frac{-v+1}{2}, u<-2 v, u<v+2\right\} .
$$

Thus each point $\mathbf{r} \in \mathbb{R}^{2}$ in this triangle shares a common period $\pi$. This gives the most left cutout triangle in Figure 1. 
Thus $\mathcal{D}_{d}^{0}$ emerges from the known set $\mathcal{D}_{d}$ by cutting out polyhedra. We will next show that $D_{d}^{0}$ is Lebesgue measurable. We use a variant of the theory on contracting polynomials which was first studied in Schur [39] and is treated more generally in Takagi [40]. Since [40] is available only in Japanese we also refer to Akiyama-Gjini [5], where the important parts of Takagi's theory are discussed in English language.

First we define the set

$$
\mathcal{E}_{d}(\rho):=\left\{\left(r_{1}, \ldots, r_{d}\right) \in \mathbb{R}^{d} \mid X^{d}+r_{d} X^{d-1}+\cdots+r_{1} \text { has only roots } y \in \mathbb{C} \text { with }|y|<\rho\right\} .
$$

Proposition 4.9 (Schur, Takagi). Let $0<\rho \leq 1$. The zeros of the polynomial

$$
g(X)=X^{n}+a_{n-1} X^{n-1}+\cdots+a_{1} X+a_{0} \in \mathbb{R}[X]
$$

are all contained in the disc $|X|<\rho$ if and only if the Hermitian form

$$
\begin{aligned}
H_{n}\left(X_{0}, X_{1}, \ldots, X_{n-1}\right):= & \sum_{\ell=0}^{n-1}\left|\rho^{n} X_{\ell}+\rho^{n-1} a_{n-1} X_{\ell+1}+\cdots+\rho^{\ell+1} a_{\ell+1} X_{n-1}\right|^{2} \\
& -\sum_{\ell=0}^{n-1}\left|a_{0} X_{\ell}+\rho a_{1} X_{\ell+1}+\cdots+\rho^{n-1-\ell} a_{n-1-\ell} X_{n-1}\right|^{2}
\end{aligned}
$$

is positive definite $\left(a_{n}=1\right)$, which is equivalent to

$$
\delta_{\nu}(\rho)>0 \quad(0 \leq \nu \leq n-1)
$$

where

$$
\delta_{\nu}(\rho)=\operatorname{det}\left(\begin{array}{cccccccc}
\rho^{n} & 0 & \ldots & 0 & a_{0} & \rho a_{1} & \ldots & \rho^{\nu} a_{\nu} \\
\rho^{n-1} a_{n-1} & \rho^{n} & \ldots & 0 & 0 & a_{0} & \ldots & \rho^{\nu-1} a_{\nu-1} \\
\vdots & & \ddots & & \vdots & & \ddots & \vdots \\
\rho^{n-\nu} a_{n-\nu} & \rho^{n-\nu+1} a_{n-\nu+1} & \cdots & \rho^{n} & 0 & 0 & \ldots & a_{0} \\
a_{0} & 0 & \ldots & 0 & \rho^{n} & \rho^{n-1} a_{n-1} & \ldots & \rho^{n-\nu} a_{n-\nu} \\
\rho a_{1} & a_{0} & \ldots & 0 & 0 & \rho^{n} & \ldots & \rho^{n-\nu+1} a_{n-\nu+1} \\
\vdots & & \ddots & & \vdots & & \ddots & \vdots \\
\rho^{\nu} a_{\nu} & \rho^{\nu-1} a_{\nu-1} & \cdots & a_{0} & 0 & 0 & \cdots & \rho^{n}
\end{array}\right) .
$$

Proof. This is an immediate consequence of Schur [39, Satz XVII].

Theorem 4.10. The sets $\mathcal{D}_{d}$ and $\mathcal{D}_{d}^{0}$ are Lebesgue measurable for each $d \in \mathbb{N}$. If $\lambda$ denotes the $d$-dimensional Lebesgue measure then $\lambda\left(\mathcal{D}_{d}\right)=\lambda\left(\mathcal{E}_{d}(1)\right)$.

Proof. By using Lemma 4.1 and $4.2, \mathcal{E}_{d}(1) \subset \mathcal{D}_{d} \subset \overline{\mathcal{E}_{d}(1)}$. Since $\mathcal{E}_{d}(1)$ is a bounded set in $\mathbb{R}^{d}$ given by polynomial inequalities $\delta_{\nu}(1)>0$ for $\nu=1, \ldots, d$, the boundary of $\mathcal{E}_{d}(1)$ has measure zero by $d$-dimensional Lebesgue measure. (Polynomials are measurable functions.) Thus $\mathcal{D}_{d}$ is Lebesgue measurable and has the same Lebesgue measure as $\mathcal{E}_{d}(1)$. Using (4.6) we now see that also $\mathcal{D}_{d}^{0}$ is Lebesgue measurable.

Remark 4.11. By the same reasoning we can easily see that the periodic points of $\tau_{P}$ exist for all polynomials $P$, whose coefficient vectors lie in an analogously defined polyhedron. However, $\mathcal{C}_{d}$ is countable. So we know in advance that its $d$-dimensional Lebesgue measure is zero. Again we get a representation of the shape

$$
\mathcal{C}_{d}^{0}=\mathcal{C}_{d} \backslash \bigcup_{\pi \neq 0}\left(\mathcal{P}^{\prime}(\pi) \cap \mathbb{Z}^{d}\right)
$$

Here the polyhedron $\mathcal{P}^{\prime}(\pi)$ is defined in terms of $T_{P}$ in an analogous way as $\mathcal{P}^{\prime}(\pi)$ was defined in terms of $\tau_{\mathbf{r}}$. 


\section{Algorithms FOR Shift RADiX SYStems}

In general it is easier to examine that a given vector $\mathbf{r}$ does not belong to $\mathcal{D}_{d}^{0}$ by producing a periodic element than examining that $\mathbf{r} \in \mathcal{D}_{d}^{0}$. However, the following result provides a very efficient algorithm which allows one to decide whether a given vector $\mathbf{r}$ is an element of $\mathcal{D}_{d}^{0}$. It is a generalization of an analogous result of Brunotte [11] on the mapping $\tau_{P}$.

Theorem 5.1. Suppose that there exists a set $\mathcal{V} \subset \mathbb{Z}^{d}$ satisfying

(i) $\mathcal{V}$ contains $2 d$ elements of the form $(0, \ldots, 0, \pm 1,0, \ldots, 0)$.

(ii) $\tau_{\mathbf{r}}(\mathcal{V}) \cup \tau_{\mathbf{r}}^{*}(\mathcal{V}) \subset \mathcal{V}$, where $\tau_{\mathbf{r}}^{*}(\mathbf{x})=-\tau_{\mathbf{r}}(-\mathbf{x})$.

(iii) For each $\mathbf{a} \in \mathcal{V}$ there is some $k>0$ such that $\tau_{\mathbf{r}}^{k}(\mathbf{a})=0$.

Then $\mathbf{r} \in \mathcal{D}_{d}^{0}$.

The following proof is similar to the proof of Lemma 4.1 in Akiyama-Rao [7].

Proof. Put $\mathbf{r}=\left(r_{1}, \ldots, r_{d}\right)$ and $r_{d+1}=1$. We say that $\mathbf{a} \in \mathbb{Z}^{d}$ has an SRS expansion if there exist some $k>0$ that $\tau_{\mathbf{r}}^{k}(\mathbf{a})=0$. Suppose that $\mathbf{a}=\left(a_{1}, \ldots, a_{d}\right)$ has an SRS expansion and $\mathbf{b}=\left(b_{1}, \ldots, b_{d}\right) \in \mathcal{V}$, and we wish to show that $\mathbf{a}+\mathbf{b}$ has an SRS expansion. Denote by $\tau_{\mathbf{r}}(\mathbf{a})=$ $\left(a_{2}, a_{3}, \ldots, a_{d+1}\right)$ and $\tau_{\mathbf{r}}(\mathbf{b})=\left(b_{2}, b_{3}, \ldots, b_{d+1}\right)$. Of course, $a_{d+1}=-\lfloor\mathbf{r a}\rfloor$ and $b_{d+1}=-\lfloor\mathbf{r b}\rfloor$. Then we have

and

$$
0 \leq r_{1} a_{1}+r_{2} a_{2}+\cdots+r_{d+1} a_{d+1}<1
$$

Thus we have

$$
0 \leq r_{1} b_{1}+r_{2} b_{2}+\cdots+r_{d+1} b_{d+1}<1 .
$$

$$
0 \leq \xi=r_{1}\left(a_{1}+b_{1}\right)+r_{2}\left(a_{2}+b_{2}\right)+\cdots+r_{d+1}\left(a_{d+1}+b_{d+1}\right)<2 .
$$

Suppose first that $\xi<1$. Then we have

$$
\tau_{\mathbf{r}}(\mathbf{a}+\mathbf{b})=\left(a_{2}+b_{2}, \ldots, a_{d+1}+b_{d+1}\right) .
$$

This means $\tau_{\mathbf{r}}(\mathbf{a}+\mathbf{b})=\tau_{\mathbf{r}}(\mathbf{a})+\tau_{\mathbf{r}}(\mathbf{b})$. Now suppose that $\xi \geq 1$. Then we see

$$
0 \leq \xi-1=r_{1}\left(a_{1}+b_{1}\right)+\cdots+r_{d}\left(a_{d}+b_{d}\right)+r_{d+1}\left(a_{d+1}+b_{d+1}-1\right)<1
$$

and

$$
\tau_{\mathbf{r}}(\mathbf{a}+\mathbf{b})=\left(a_{2}+b_{2}, \ldots, a_{d}+b_{d}, a_{d+1}+b_{d+1}-1\right) .
$$

Noting that in this case

$$
0<r_{1} b_{1}+r_{2} b_{2}+\cdots+r_{d+1} b_{d+1}<1,
$$

holds, we have

$$
0<r_{1}\left(-b_{1}\right)+\cdots+r_{d}\left(-b_{d}\right)+r_{d+1}\left(-b_{d+1}+1\right)<1,
$$

which implies $\tau_{\mathbf{r}}(\mathbf{a}+\mathbf{b})=\tau_{\mathbf{r}}(\mathbf{a})+\tau_{\mathbf{r}}^{*}(\mathbf{b})$. Repeating this argument, we have for any $n$,

$$
\tau_{\mathbf{r}}^{n}(\mathbf{a}+\mathbf{b})=\tau^{n}(\mathbf{a})+\eta
$$

for some $\eta \in \mathcal{V}$. Since $\mathbf{a}$ has an SRS expansion, $\tau_{\mathbf{r}}^{n}(\mathbf{a}+\mathbf{b}) \in \mathcal{V}$ for a large $n$. Now from assumption (iii), we conclude that $\mathbf{a}+\mathbf{b}$ has an SRS expansion. By assumption (i), it is now easy to get the result.

Assume that the spectral radius of $R(\mathbf{r})$ is less than 1 . Then Theorem 5.1 gives an efficient algorithm to determine whether $\mathbf{r} \in \mathcal{D}_{d}^{0}$ or not. In fact, let $\mathcal{V}_{1}$ is the set of $2 d$ elements of the form $(0, \ldots, 0, \pm 1,0, \ldots, 0)$ and define inductively

$$
\mathcal{V}_{i+1}=\mathcal{V}_{i} \cup \tau_{\mathbf{r}}\left(\mathcal{V}_{i}\right) \cup \tau_{\mathbf{r}}^{*}\left(\mathcal{V}_{i}\right)
$$

As $\tau_{\mathbf{r}}$ and $\tau_{\mathbf{r}}^{*}$ are contractive on the lattice $\mathbb{Z}^{d}$, there must exist $i$ that $\mathcal{V}_{i+1}=\mathcal{V}_{i}=\mathcal{V}$. Thus it suffices to check if the elements of this $\mathcal{V}$ satisfy the last condition or not. This can be tested in a finite amount of time by Lemma 4.2. We shall call this set $\mathcal{V}$ a set of witnesses. The set of witnesses was first introduced in the CNS case in [11] and [36] independently. In [8], a similar algorithm was introduced in the $\beta$-expansion setting. It turned out by using SRS, there is no need to consider these problems separately. This is one of the considerable advantages of SRS. 
The following result gives a generalization of Theorem 5.1 which gives an efficient way to determine a subregion of $\mathcal{D}_{d}^{0}$ contained in a given convex polyhedron lying in the interior of $\mathcal{D}_{d}$.

Theorem 5.2. Let $\mathbf{r}_{1}, \ldots, \mathbf{r}_{k}$ be points of $\mathcal{D}_{d}$ and denote by $H$ the convex hull of $\mathbf{r}_{1}, \ldots, \mathbf{r}_{k}$. We assume that $H$ is contained in the interior of $\mathcal{D}_{d}$ and is sufficiently small in diameter. For $\mathbf{z} \in \mathbb{Z}^{d}$ take $M(\mathbf{z})=\max _{1 \leq i \leq k}\left\{-\left\lfloor\mathbf{r}_{i} \mathbf{z}\right\rfloor\right\}$. Then there exists an algorithm to create a finite directed graph $(V, E)$ with vertices $V \subset \mathbb{Z}^{d}$ and edges $E \in V \times V$ which satisfy

(1) each d-dimensional standard unit vector $(0, \ldots, 0, \pm 1,0, \ldots, 0) \in V$,

(2) for each $\mathbf{z}=\left(z_{1}, \ldots, z_{d}\right) \in V$ and

$$
j \in[-M(-\mathbf{z}), M(\mathbf{z})] \cap \mathbb{Z}
$$

we have $\left(z_{2}, \ldots, z_{d}, j\right) \in V$ and a directed edge $\left(z_{1}, \ldots, z_{d}\right) \rightarrow\left(z_{2}, \ldots, z_{d}, j\right)$ in $E$.

(3) $H \cap \mathcal{D}_{d}^{0}=H \backslash \bigcup_{\pi} P(\pi)$, where $\pi$ are taken over all nonzero primitive cycles of $(V, E)$.

Proof. For $\mathbf{z} \in \mathbb{Z}^{d}$ the set $I(\mathbf{z})=\{\mathbf{s z} \mid \mathbf{s} \in H\}$ is an interval in $\mathbb{R}$ since the linear image of a convex set must be convex. It is easily seen that its end points must be of the form $\mathbf{r}_{i} \mathbf{z}$ for some $i$. Set $m(\mathbf{z})=\min _{1 \leq i \leq k}\left\{-\left\lfloor\mathbf{r}_{i} \mathbf{z}\right\rfloor\right\}$. Then, since $-M(-\mathbf{z}) \leq m(\mathbf{z})$ and $M(\mathbf{z}) \geq-m(-\mathbf{z})$, we have

$$
-M(-\mathbf{z})=\min \{m(\mathbf{z}),-M(-\mathbf{z})\} \leq-\lfloor\mathbf{s z}\rfloor,\lfloor-\mathbf{s z}\rfloor \leq \max \{-m(-\mathbf{z}), M(\mathbf{z})\}=M(\mathbf{z}) .
$$

Since $H \subset \operatorname{int}\left(\mathcal{D}_{d}\right)$, Lemmas 4.1 and 4.3 imply the existence of a positive $\delta<1$ such that for each $\mathbf{r} \in H$, the spectral radius of the matrix $R(\mathbf{r})$ is less than $\delta$. Let $V_{1}$ be the set of $d$-dimensional standard unit vectors $(0, \ldots, 0, \pm 1,0, \ldots, 0)$ and inductively define

$$
V_{i+1}=V_{i} \cup\left\{\left(z_{2}, \ldots, z_{d}, j\right) \mid \mathbf{z}=\left(z_{1}, \ldots, z_{d}\right) \in V_{i} \text { and } j \in[-M(-\mathbf{z}), M(\mathbf{z})] \cap \mathbb{Z}\right\} .
$$

As $I(\mathbf{z})$ is an interval, we can find $\mathbf{r} \in H$ such that $\left(z_{2}, \ldots, z_{d}, j\right)^{t}=R(\mathbf{r})\left(z_{1}, \ldots, z_{d}\right)^{t}+(0, \ldots, 0, v)^{t}$ with $|v|<1$. Thus the maps $\left(z_{1}, \ldots, z_{d}\right) \rightarrow\left(z_{2}, \ldots, z_{d}, j\right)$ are uniformly contractive on the lattice $\mathbb{Z}^{d}$ provided $H$ is sufficiently small in diameter. Indeed, we start with a point $\mathbf{r} \in H$ and choose a norm such that $R(\mathbf{r})$ is contractive. Then for a suffciently small neighborhood of $r$, the maps $\left(z_{1}, \ldots, z_{d}\right) \rightarrow\left(z_{2}, \ldots, z_{d}, j\right)$ are contractive with respect to this norm. Thus there exists $i$ with $V_{i+1}=V_{i}$. Let $V=V_{i}$ and define the edges $E$ by $(2)$. Then it is clear that $(V, E)$ is a finite directed graph with (1) and (2).

We shall show (3). It is obvious that $H \backslash \bigcup_{\pi} P(\pi) \supset H \cap \mathcal{D}_{d}^{0}$. Let $\mathbf{s} \in H$, which is an inner point of $D_{d}$. Following the construction after Theorem 5.1, we define a set of vertices $\mathcal{V}$ which corresponds to a single point $\mathbf{s}$. Denote by $\mathcal{E}$ the set of edges defined in the following way. There exists an edge from $v_{1} \in \mathcal{V}$ to $v_{2} \in \mathcal{V}$ if and only if $v_{2}=\tau_{\mathbf{s}}\left(v_{1}\right)$ or $v_{2}=\tau_{\mathbf{s}}^{*}\left(v_{1}\right)$. The construction of $V$ in (5.2) implies that $\mathcal{V} \subset V$. Thus (5.1) implies that $(\mathcal{V}, \mathcal{E})$ is a subgraph of $(V, E)$. If $\mathbf{s} \in \mathcal{D}_{d} \backslash \mathcal{D}_{d}^{0}$ then by Theorem 5.1 there exists a non zero primitive cycle $\pi$ in the graph $(\mathcal{V}, \mathcal{E})$ and $\mathbf{s} \in P(\pi)$. This proves the theorem since $\pi$ is a nonzero primitive cycle of the graph $(V, E)$ as well.

Note that this theorem gives an effective algorithm to obtain $H \cap \mathcal{D}_{d}^{0}$ for any compact set $H$ lying in the interior of $\mathcal{D}_{d}$. In fact, firstly as $H$ is compact and contained in the interior of $\mathcal{D}_{d}$, there exists a finite covering of $H$ by sufficiently small convex polyhedra each of which is contained in the interior of $\mathcal{D}_{d}$. Secondly, the number of primitive cycles of a finite directed graph must be finite.

In practice, to apply this theorem, there is no need to care of the "sufficiently small" condition. Just choose a small convex hull and try this algorithm to see whether the emerging process terminates or not in a certain prescribed time. If it terminates, the argument works and we get $H \cap \mathcal{D}_{d}^{0}$. If not, then we try again by a smaller convex hull.

Especially the following case appears often in practice.

Corollary 5.3. If $(V, E \backslash\{0 \rightarrow 0\})$ is a tree, then $H \subset \mathcal{D}_{d}^{0}$.

Theorem 5.2 also implies

Corollary 5.4. Let $d \in \mathbb{N}$ and $M$ be a closed set contained in the interior of $\mathcal{D}_{d}$. Then $\mathcal{D}_{d}^{0} \cap M$ can be characterized by cutting out finitely many polyhedra from $\mathcal{D}_{d} \cap M$. 
Let us give a few remark on computational issues. The algorithm of Brunotte in [11] for CNS slightly differs from the one in this paper. Indeed, his initial set $\mathcal{V}_{1}$ was a single point $(1,0, \ldots, 0)$. Unfortunately the final sets $\mathcal{V}$ are usually the same by this improvement which is valid for CNS. ${ }^{1}$

Borbély [10] described an optimized implementation of the algorithm based on this smaller initial set. He computed $\mathcal{C}_{3}^{0}$ for all $\left(p_{0}, p_{1}, p_{2}\right)$ with $p_{0} \leq 474$.

To show how hard it is to decide whether a polynomial belongs to $\mathcal{C}_{d}^{0}$ we give two examples: For $X^{8}+2 X^{7}+3 X^{6}+3 X^{5}+3 X^{4}+3 X^{3}+3 X^{2}+3 X+2$ the smallest set of witnesses has 241,719 elements (cf. A. Kovács [22]), while for $X^{3}+317 X^{2}+632 X+317$ it has 1, 308, 322 elements.

A natural question is whether the CNS property belongs to the NP or to the coNP class. The above examples indicate that the CNS property cannot be decided in polynomial time.

However, if the polynomial $P(X)$ fulfills certain conditions, it has been shown that there exist relatively small sets of witnesses (cf. for instance Akiyama and Pethő [6] as well as Scheicher and Thuswaldner [36]).

\section{LIFTING AND LONG PERIODS}

Let $\pi$ be a period of $\mathcal{C}_{d}$ or $\mathcal{D}_{d}$ which corresponds to a non-degenerate cutout polyhedron (i.e. a cutout polyhedron with positive $d$-dimensional Lebesgue measure). Then we call $\pi$ a nondegenerate period. In this section we will show that we can "lift" a non-degenerate period to higher dimensions.

\section{Definition 6.1. Let}

$$
\pi:\left(a_{1}, \ldots, a_{d}\right) ; a_{d+1}, \ldots, a_{L}
$$

be a non-degenerate period of length $L$ of $\mathcal{C}_{d}$ or $\mathcal{D}_{d}$. Then we call

$$
l(\pi):\left(a_{1}, a_{2}, \ldots, a_{d+1}\right) ; a_{d+2}, \ldots, a_{L}
$$

the lift of $\pi$ to $d+1$.

Note that $\pi$ and $l(\pi)$ have the same period length $L$. The main result of this section is the following theorem.

Theorem 6.2 (Lifting Theorem). Let $d \geq 1$ be an integer.

- Let $p_{0} \geq 2$ and let $\pi$ be a non-degenerate period for $\mathcal{C}_{d}$. Then $\pi$ is also a non-degenerate period for $\mathcal{D}_{d}$. More precisely, there exist $p_{1}, \ldots, p_{d-1} \in \mathbb{Z}$ such that $\left(p_{0}, \ldots, p_{d-1}\right) \in$ $\operatorname{int}\left(\mathcal{P}^{\prime}(\pi)\right)$ and

$$
\left(\frac{1}{p_{0}}, \frac{p_{d-1}}{p_{0}}, \cdots, \frac{p_{1}}{p_{0}}\right) \in \operatorname{int}(\mathcal{P}(\pi))
$$

- Let $\pi$ be a non-degenerate period of $\mathcal{D}_{d}$. Then its lift $\lambda:=l(\pi)$ is a non-degenerate period of $\mathcal{C}_{d+1}$ for each sufficiently large $p_{0}$. More precisely, for all $\left(r_{1}, \ldots, r_{d}\right) \in \operatorname{int}(\mathcal{P}(\pi))$ there exists $\varepsilon>0$ such that for all $\left(p_{0}, \ldots, p_{d}\right) \in \mathbb{Z}^{d+1}$ with

$$
\max _{1 \leq k \leq d}\left|\frac{p_{d+1-k}}{p_{0}}-r_{k}\right|<\varepsilon
$$

we have $\left(p_{0}, \ldots, p_{d}\right) \in \operatorname{int}\left(\mathcal{P}^{\prime}(\lambda)\right)$.

Proof. Let $\pi$ be a non-degenerate period of $\mathcal{C}_{d}$ of shape (6.1). Then for sufficiently large $p_{0}$ there exists $P(X)=X^{d}+p_{d-1} X^{d-1}+\cdots+p_{0} \in \mathbb{Z}[X]$ such that $\left(p_{0}, \ldots, p_{d-1}\right)$ is an inner point of the cutout polyhedron defined by $\pi$. This implies that

$$
\begin{aligned}
& 0<a_{j+1}+p_{d-1} a_{j+2}+\cdots+p_{1} a_{j+d}+p_{0} a_{j+d+1}<p_{0} \quad \text { or equivalently } \\
& 0<\frac{1}{p_{0}} a_{j+1}+\frac{p_{d-1}}{p_{0}} a_{j+2}+\cdots+\frac{p_{1}}{p_{0}} a_{j+d}+a_{j+d+1}<1
\end{aligned}
$$

\footnotetext{
${ }^{1}$ We could not find an example that the final sets are different.
} 
holds for all $0 \leq j \leq L-1$. But by the definition of $\tau_{\mathbf{r}}$ this implies that $\tau_{\mathbf{r}}$ has the period $\pi$ for

$$
\mathbf{r}=\left(\frac{1}{p_{0}}, \frac{p_{d-1}}{p_{0}}, \cdots, \frac{p_{1}}{p_{0}},\right)
$$

Since the inequalities are strict we even see that the corresponding cutout polyhedron is nondegenerate. This proves the first assertion.

In order to prove the second assertion assume that $\pi$ is a non-degenerate period of $\mathcal{D}_{d}$. Then there exists an $\mathbf{r}=\left(r_{1}, \ldots, r_{d}\right)$ such that

$$
0<r_{1} a_{j+1}+r_{2} a_{j+2}+\cdots+r_{d} a_{j+d}+a_{j+d+1}<1
$$

holds for $0 \leq j \leq L-1$. If $p_{0}$ is large enough we can find, by Dirichlet's approximation theorem, rational approximations $p_{d+1-k} / p_{0}$ to $r_{k}(1 \leq k \leq d)$ such that

$$
0<\frac{p_{d}}{p_{0}} a_{j+1}+\frac{p_{d-1}}{p_{0}} a_{j+2}+\cdots+\frac{p_{1}}{p_{0}} a_{j+d}+a_{j+d+1}<1
$$

hold for $0 \leq j \leq L-1$. For large $p_{0}$ we can even add $a_{j} / p_{0}$ without violating the inequalities. This means that

$$
\begin{aligned}
& 0<\frac{1}{p_{0}} a_{j}+\frac{p_{d}}{p_{0}} a_{j+1}+\cdots+\frac{p_{1}}{p_{0}} a_{j+d}+a_{j+d+1}<1 \text { or equivalently } \\
& 0<a_{j}+p_{d} a_{j+1}+\cdots+p_{1} a_{j+d}+p_{0} a_{j+d+1}<p_{0}
\end{aligned}
$$

holds for $(1 \leq j \leq L)$. By the definition of $\tau_{P}$ we conclude from these chains of inequalities that $\tau_{P}$ has the period $l(\pi)$. Since all inequalities are strict we even see that the corresponding cutout polyhedron is non-degenerate and we are ready.

This will permit us to "lift" results, which we can show in low dimension to higher dimensions via the chain

$$
\mathcal{D}_{2} \longrightarrow \mathcal{C}_{3} \longrightarrow \mathcal{D}_{3} \longrightarrow \mathcal{C}_{4} \longrightarrow \cdots \longrightarrow \mathcal{C}_{d} \longrightarrow \mathcal{D}_{d} \longrightarrow \mathcal{C}_{d+1} \longrightarrow \cdots
$$

Despite it is very easy this will be helpful. We will use it now in order to show that in $\mathcal{C}_{d}$ and $\mathcal{D}_{d}$ there exist arbitrarily long periods giving rise to non-degenerate polyhedra (non-degenerate periods, for short).

Consider the following family of edges.

$$
\begin{array}{rrrrrr}
\alpha_{k}: & (-k-1,-n+k) & \rightarrow & (-n+k, k+1) & & (0 \leq k \leq n-1), \\
\beta_{k}: & (-n+k, k+1) & \rightarrow & (k+1, n+1-k) & (0 \leq k \leq n-1), \\
\gamma_{0}: & (1, n+1) & \rightarrow & (n+1,1), & \\
\gamma_{k}: & (k+1, n+1-k) & \rightarrow & (n+1-k,-k) \\
\gamma_{n}: & (n+1,1) & \rightarrow & (1,-n), & \\
\delta_{k}: & (n+1-k,-k) & \rightarrow & (-k,-n-1+k) & & (1 \leq k \leq n) .
\end{array}
$$

With these edges we form the cycle

$$
\zeta_{n}: \quad \alpha_{0} \beta_{0} \gamma_{0} \gamma_{n} \delta_{n} \alpha_{n-1} \beta_{n-1} \gamma_{n-1} \delta_{n-1} \alpha_{n-2} \ldots \alpha_{1} \beta_{1} \gamma_{1} \delta_{1}
$$

Note that $\delta_{1}$ ends up in $(-1,-n)$. In this node $\alpha_{0}$ starts. Thus $\zeta_{n}$ is indeed a cycle. We wonder whether there exists $\mathbf{r}:=\left(x_{n}, y_{n}\right) \in \mathcal{D}_{2}$ such that $\tau_{\mathbf{r}}$ has $\zeta_{n}$ as a non-degenerate period. This is done in the following result.

Theorem 6.3. Fix $n \in \mathbb{N}, n>3$, and set $\mathbf{r}=\left(x_{n}, y_{n}\right) \in \mathbb{R}^{2}$ with

$$
x_{n}:=1-\frac{1}{2 n^{2}}+z_{n} \quad \text { and } \quad y_{n}:=-\frac{2 n+1}{2 n(n+1)}+u_{n},
$$

where $\left|z_{n}\right|,\left|u_{n}\right|<1 / n^{4}$. Then $\zeta_{n}$ is a non-degenerate period of $\tau_{\mathbf{r}}$.

Remark 6.4. Since we can select $n$ arbitrarily large and the length of the period $\zeta_{n}$ is $4 n+1$ the previous theorem implies that there exist non-degenerate periods of arbitrarily large length for $\mathcal{D}_{2}$. Note that each of these periods cuts out a polyhedron of the set $\mathcal{D}_{2}^{0}$. Roughly speaking, the cycle $\zeta_{n}$ is the $n$-th polyhedral cutout on the left hand side of the picture of our approximation of $\mathcal{D}_{2}^{0}$ (cf. Figure 1). 
Proof. By the definition of $\tau_{\mathbf{r}}$ we only have to show that for each edge $\left(\varepsilon_{1}, \varepsilon_{2}\right) \rightarrow\left(\varepsilon_{2}, \varepsilon_{3}\right)$ in $\zeta_{n}$ the inequalities

$$
0<x_{n} \varepsilon_{1}+y_{n} \varepsilon_{2}+\varepsilon_{3}<1
$$

are fulfilled. We start with the edges $\alpha_{k}(0 \leq k \leq n-1)$. We have to show that

$$
0<x_{n}(-k-1)+y_{n}(-n+k)+k+1<1 .
$$

In this case we can even prove that

$$
\frac{3}{2 n}-\frac{1}{2 n^{2}}-\frac{2}{n^{3}}<x_{n}(-k-1)+y_{n}(-n+k)+k+1<1-\frac{1}{2 n}+\frac{1}{n^{2}}+\frac{2}{n^{3}} .
$$

Indeed, inserting the values of $x_{n}$ and $y_{n}$ in (6.3) we see that these inequalities read

$$
\frac{3}{2 n}-\frac{1}{2 n^{2}}-\frac{2}{n^{3}}<1-\frac{k}{2 n}-\frac{k+1}{2(n+1)}+\frac{1+k}{2 n^{2}}-z_{n}(k+1)+u_{n}(k-n)<1-\frac{1}{2 n}+\frac{1}{n^{2}}+\frac{2}{n^{3}} .
$$

If $k$ is in the permitted range they are easily seen to be true. For $\beta_{k}(0 \leq k \leq n-1)$ we have to show

Here we even prove that

$$
0<x_{n}(-n+k)+y_{n}(k+1)+n+1-k<1 .
$$

$$
\begin{aligned}
\frac{1}{2 n}-\frac{2}{n^{3}} & <x_{n}(-n+k)+y_{n}(k+1)+n+1-k<1-\frac{1}{2 n}+\frac{1}{2 n^{2}}+\frac{2}{n^{3}} \Leftrightarrow \\
\frac{1}{2 n}-\frac{2}{n^{3}} & <1-\frac{k}{2 n}-\frac{k+1}{2(n+1)}-\frac{k}{2 n^{2}}+z_{n}(k-n)+u_{n}(k+1)<1-\frac{1}{2 n}+\frac{1}{2 n^{2}}+\frac{2}{n^{3}} .
\end{aligned}
$$

The latter chain of inequalities being true if $k$ is in the indicated range. For $\gamma_{0}$ we have

$$
0<x_{n}+y_{n}(n+1)+1<1 \Leftrightarrow 0<1-\frac{1}{2 n}-\frac{1}{2 n^{2}}+z_{n}+u_{n}(n+1)<1,
$$

which is valid. For $\gamma_{k}(1 \leq k \leq n-1)$ we have to show that $0<x_{n}(k+1)+y_{n}(n+1-k)-k<1$. The range of $k$ assures that even

$$
\begin{aligned}
& \frac{1}{2 n}-\frac{3}{2 n^{2}}-\frac{2}{n^{3}}<x_{n}(k+1)+y_{n}(n+1-k)-k<1-\frac{5}{2 n}+\frac{1}{n^{2}}+\frac{3}{n^{3}} \Leftrightarrow \\
& \frac{1}{2 n}-\frac{3}{2 n^{2}}-\frac{2}{n^{3}}<\frac{k-1}{2 n}+\frac{k}{2(n+1)}-\frac{k+1}{2 n^{2}}+z_{n}(k+1)+u_{n}(n+1-k)<1-\frac{5}{2 n}+\frac{1}{n^{2}}+\frac{3}{n^{3}} .
\end{aligned}
$$

holds. $\gamma_{n}$ yields

$$
0<x_{n}(n+1)+y_{n}-n<1 \Leftrightarrow 0<1-\frac{1}{2 n}-\frac{1}{n+1}-\frac{1}{2 n^{2}}-\frac{1}{2 n(n+1)}+z_{n}(n+1)+u_{n}<1
$$

and finally, $\delta_{k}(1 \leq k \leq n)$ is also fine because

$$
\begin{aligned}
& \frac{1}{2 n}+\frac{1}{2 n^{2}}-\frac{2}{n^{3}}<x_{n}(n+1-k)-y_{n} k-n-1+k<1-\frac{1}{2 n}+\frac{1}{n^{2}}+\frac{2}{n^{3}} \Leftrightarrow \\
& \frac{1}{2 n}+\frac{1}{2 n^{2}}-\frac{2}{n^{3}}<\frac{n+1-k}{2 n^{2}}+\frac{k}{n}-\frac{k}{2 n(n+1)}+z_{n}(n+1-k)-k u_{n}<1-\frac{1}{2 n}+\frac{1}{n^{2}}+\frac{2}{n^{3}}
\end{aligned}
$$

is true.

Remark 6.5. If we draw the points of the periods $\zeta_{n}$ in the plane, we get a nice "rhomboid" shape. An example is drawn in Figure 3.

The existence of long periods for $\mathcal{D}_{2}$ yields without any additional work an explicit family of arbitrarily long non-degenerate periods for each $\mathcal{D}_{d}$ with $d \geq 2$.

Theorem 6.6. Let $d \geq 2$ be an integer, fix $n \in \mathbb{N}, n>3$. Then there exist some $\mathbf{r} \in \mathbb{R}^{d}$ such that $l^{d-2}\left(\zeta_{n}\right)$ is a non-degenerate period of $\tau_{\mathbf{r}}$. Since we can select $n$ arbitrarily large and the length of the period $l^{d-2}\left(\zeta_{n}\right)$ is $4 n+1$ this implies that there exist non-degenerate periods of arbitrarily large length for $\mathcal{D}_{d}$ and $\mathcal{C}_{d+1}$.

Proof. This is a direct application of the Lifting Theorem.

We conclude this section with the following easy result. 


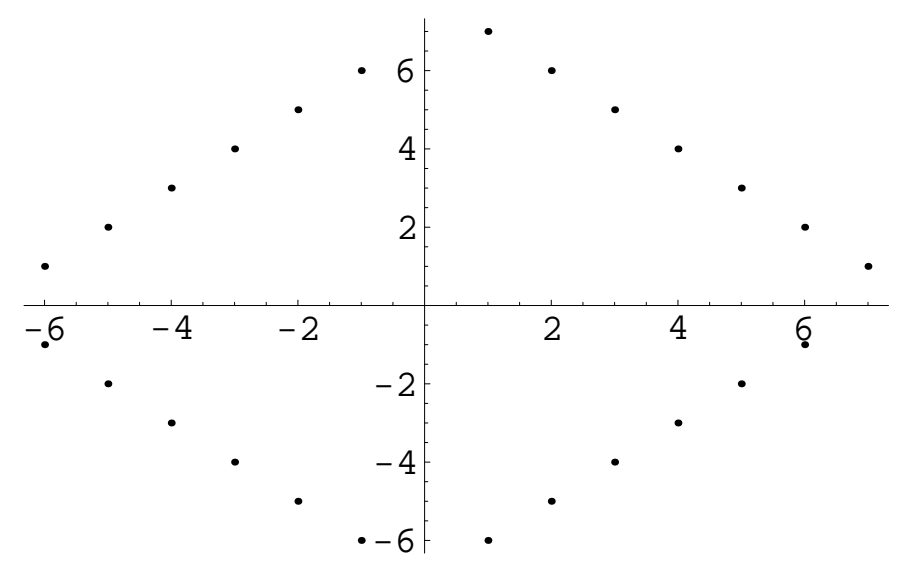

Figure 3 . The points of the period $\zeta_{6}$

Corollary 6.7. Fix $n \in \mathbb{N}, n>3$, and set $d \geq 2$ and $\mathbf{r}=\left(0, \ldots, 0, x_{n}, y_{n}\right) \in \mathbb{R}^{d}$ with $x_{n}, y_{n}$ as in Theorem 6.3. Then $l^{d-2}\left(\zeta_{n}\right)$ is a period of $\tau_{\mathbf{r}}$.

Proof. Since only the last two coordinates of $\mathbf{r}$ are nonzero this can be shown by copying the proof of Theorem 6.3 verbatim.

Remark 6.8. Note that the Lifting Theorem was essential in Theorem 6.6 in order to guarantee that the constructed periods are non-degenerate.

\section{CRitical POints}

At the end of Section 4 we observed that $\mathcal{C}_{d}^{0}$ as well as $\mathcal{D}_{d}^{0}$ can be described by cutting out countably many polyhedra from a known set. We give the following definition.

Definition 7.1. Let $\mathbf{x} \in \mathcal{D}_{d}$.

- If there exists an open neighborhood of $\mathbf{x}$ which contains only finitely many cutout polyhedra then we call $\mathbf{x}$ a regular point.

- If each open neighborhood of $\mathbf{x}$ has nonempty intersection with infinitely many cutout polyhedra then we call $\mathbf{x}$ a weak critical point for $\mathcal{D}_{d}$.

- If for each open neighborhood $U$ of $\mathbf{x}$ the set $U \backslash \mathcal{D}_{d}^{0}$ can not be covered by finitely many cutout polyhedra then $\mathbf{x}$ is called a critical point.

An analogous definition can not be given for $\mathcal{C}_{d}$ since $\mathcal{C}_{d}$ is a discrete set. Thus we confine ourselves to $\mathcal{D}_{d}$ in the following investigations.

Obviously, each critical point is a weak critical point.

Suppose that $\mathbf{x}$ is a regular point. Then there exists a neighborhood of $\mathbf{x}$ containing only finitely many cutout polyhedra. Thus the set $\mathcal{D}_{d}^{0}$ is characterized in this neighborhood by cutting out these finitely many polyhedra. This is a case which we expect to be easy.

Suppose that $\mathbf{x}$ a weak critical point. Then we can select among the infinitely many polyhedra near $\mathbf{x}$ a finite subcollection, which allows us to determine the shape of $\mathcal{D}_{d}^{0}$ near $\mathbf{x}$.

The worst case occurs if $\mathbf{x}$ is a critical point. If we can find such a point we have proved that $\mathcal{D}_{d}^{0}$ can not be obtained by cutting out finitely many polyhedra from $\mathcal{D}_{d}$. Even for the characterization of $\mathcal{D}_{d}^{0}$ in a neighborhood of $\mathbf{x}$ we need to consider infinite families of cutout polyhedra.

Sloppily spoken, near a weak critical point there are many cutout polyhedra. Near a critical point there are many non-overlapping cutout polyhedra.

The aim of the present section is to prove the existence of critical points for each $d \geq 2$. This shows that there is no way to characterize either of the sets $\mathcal{D}_{d}^{0}$ by finitely many cutouts if $d \geq 2$.

From Section 4 the following result follows immediately.

Lemma 7.2. Let $\mathbf{x}$ be a weak critical point for $\mathcal{D}_{d}$. Then $\mathbf{x} \in \partial \mathcal{E}_{d}(1)$. 
Proof. Let $\mathbf{x}$ be an inner point of $\mathcal{E}_{d}(1)$. Then there exists an $r<1$ such that $\mathbf{x}$ is contained in the interior of $\mathcal{E}_{d}(r)$. Thus by Corollary 5.4 at most finitely many cutout polyhedra can have non-empty intersection with a sufficiently small neighborhood $U$ of $\mathbf{x}$. This shows the result.

We will now show the existence of a critical point for $\mathcal{D}_{d}$ with $d \geq 2$. This implies that the family of cutout polyhedra is infinite for the set $\mathcal{D}_{d}^{0}$ if $d \geq 2$. An application of the Lifting theorem shows that the same holds true for the sets $\mathcal{C}_{d}^{0}$ if $d \geq 3$. Recall that $\mathcal{C}_{2}^{0}$ is known explicitly. It emerges from $\mathcal{C}_{2}$ by cutting out finitely many polyhedra.

Proposition 7.3. The point $\mathbf{K}_{d}:=(0, \ldots, 0,1,0) \in \mathbb{R}^{d}$ is a weak critical point of $\mathcal{D}_{d}$.

Proof. This is just another formulation of Corollary 6.7. Just note that $\left(0, \ldots, 0, x_{n}, y_{n}\right)$ converges to $\mathbf{K}_{d}$ if $n \rightarrow \infty$.

In order to prove the existence of a critical point we have to show that the cutout polyhedra corresponding to the periods $\zeta_{n}$ can not be covered by finitely many cutout polyhedra. To this matter we need the following lemma.

Lemma 7.4. Let $\left\{x_{n}\right\}_{n \geq 1}$ and $\left\{y_{n}\right\}_{n \geq 1}$ be sequences with $x_{n}<1, y_{n}<0, \lim x_{n}=1, \lim y_{n}=0$ and $1-x_{n}=o\left(y_{n}\right)$. Let $\left\{a_{m}\right\}_{m \geq 1}$ be a sequence of integers such that $\left|a_{i}\right|<K$ for some constant $K$. Then there exists $N \in \mathbb{N}$ such that

$$
0 \leq a_{i-1} x_{n}+a_{i} y_{n}+a_{i+1}<1
$$

can not hold for all $i$ if $n \geq N$ unless $a_{i}=0$ for all $i$ large enough. Thus nonzero periods whose elements are bounded by $K$ can not occur in $\mathcal{D}_{d}$ for $\tau_{\left(0 \ldots, 0, x_{n}, y_{n}\right)}$ if $n$ is large enough.

Proof. We can rewrite (7.1) as

$$
0 \leq a_{i-1}\left(x_{n}-1\right)+a_{i} y_{n}+a_{i-1}+a_{i+1}<1 .
$$

For convenience set $d_{i}:=a_{i-1}\left(x_{n}-1\right)+a_{i} y_{n}(i \in \mathbb{N})$. This yields that

$$
a_{i-1}+a_{i+1}= \begin{cases}0, & \text { for } d_{i} \geq 0, \\ 1, & \text { for } d_{i}<0\end{cases}
$$

for large $n$. Also if we choose $n$ large enough, by $1-x_{n}=o\left(y_{n}\right)$ the case $d_{i}=0$ is excluded if $\left(a_{i-1}, a_{i}\right) \neq(0,0)$ and

$$
\operatorname{sign}\left(d_{i}\right)= \begin{cases}-\operatorname{sign}\left(a_{i}\right), & \text { for } a_{i} \neq 0, \\ -\operatorname{sign}\left(a_{i-1}\right), & \text { for } a_{i}=0\end{cases}
$$

holds. W.l.o.g. we have to show that $\left(a_{1}, a_{2}\right) \neq(0,0)$ implies that $a_{i}=0$ for all $i$ large enough. By (7.1) it even suffices to show that there is some $i \in \mathbb{N}$ such that $a_{i}=a_{i+1}=0$. To this matter we have to distinguish several cases.

$\mathrm{I} / 1$ Let $a_{1}>1$ and $a_{2}>0$.

$$
a_{1}>1, a_{2}>0 \Rightarrow d_{2}<0 \text { and, by (7.2) it follows that } a_{3}=1-a_{1}<0 .
$$

Going on this way yields

$$
\begin{aligned}
d_{3}>0 \text { and } a_{4}=-a_{2}<0 & \Longrightarrow d_{4}>0, a_{5}=-a_{3}=a_{1}-1>0 \\
& \Longrightarrow d_{5}<0, a_{6}=1-a_{4}=a_{2}+1>0 .
\end{aligned}
$$

Iterating this we easily see that for each $s \leq a_{1}-1$ we have

$$
a_{4 s+1}=a_{1}-s \quad \text { and } \quad a_{4 s+2}=a_{2}+s .
$$

Thus we can confine ourselves to $a_{1}=1$.

$\mathrm{I} / 2$ Let $a_{1}=1$ and $a_{2}>2\left(a_{2}=2\right.$ is treated later). We have, using (7.2)

$$
\begin{array}{rll}
a_{1}=1, a_{2}>2 & \Longrightarrow & d_{2}<0, a_{3}=0 \quad \Longrightarrow \\
d_{3}<0, a_{4}=1-a_{2}<0 & \Longrightarrow & d_{4}>0, a_{5}=-a_{3}=0 \quad \Longrightarrow \\
d_{5}>0, a_{6}=-a_{4}=a_{2}-1>1 & \Longrightarrow & d_{6}<0, a_{7}=1 .
\end{array}
$$


By (7.3) there exist $a_{6}>1, a_{7}>0$ with $a_{6}+a_{7}=a_{2}$. This means that we are in case I/ 1 again and after a few iterations we arrive at

$$
\left(a_{\ell}, a_{\ell+1}\right)=\left(1, a_{\ell+1}\right)
$$

with $a_{\ell+1}=a_{2}-1$. Iterating this procedure we arrive at $\mathrm{I} / 2$ after finitely many steps.

Thus the cases $\mathrm{I} / 1$ and $\mathrm{I} / 2$ are reduced to the following case.

$\mathrm{I} / 3 a_{1}=1$ and $a_{2}=2$. Starting with this constellation we see that

$$
\begin{aligned}
a_{1}=1, a_{2}=2 & \Longrightarrow d_{2}<0, a_{3}=0 \Longrightarrow \\
d_{3}<0, a_{4}=1-a_{2}=-1 & \Longrightarrow d_{4}>0, a_{5}=0 \Longrightarrow \\
d_{5}>0, a_{6}=1 & \Longrightarrow \quad d_{6}<0, a_{7}=1 \Longrightarrow \\
d_{7}<0, a_{8}=1-a_{6}=0 & \Longrightarrow \quad d_{8}<0, a_{9}=1-a_{7}=0 .
\end{aligned}
$$

Thus we end up in a zero period.

II Let now $a_{1}<0$ and $a_{2} \leq 0$. This implies that

$$
d_{2}>0, a_{3}=-a_{1}>0 \Longrightarrow d_{3}<0, a_{4}=1-a_{2}>0 .
$$

Thus we reduced this case to case I and the sequence terminates with 0 .

III Let $a_{1} \geq 0$ and $a_{2}<0$. This implies that

$$
d_{2}>0, a_{3}=-a_{1} \leq 0
$$

and we are in case II.

IV $a_{1}<0$ and $a_{2}>0$. This yields $d_{2}<0$ and $a_{3}=1-a_{1}>0$ and we are in case I.

$\mathrm{V} a_{1}=0$ and $a_{2}>0$. This yields $a_{3}=1$ and we are in case I.

VI $a_{1}>0$ and $a_{2}=0$. This yields $a_{3} \leq 0$. Thus either $a_{2}=a_{3}=0$ and we are ready or $a_{2}=0$ and $a_{3}<0$ and we are in case III.

With help of this lemma we are in a position to give the following result.

Theorem 7.5. Let $d \geq 2$. Then $\mathbf{K}_{d}:=(0, \ldots, 1,0) \in \mathbb{R}^{d}$ is a critical point of $\mathcal{D}_{d}$.

Proof. Let $U \subset \mathcal{D}_{d}$ be an arbitrary neighborhood of $\mathbf{K}_{d}$ in $\mathcal{D}_{d}$. We have to show that $U \backslash \mathcal{D}_{d}^{0}$ can not be covered with finitely many sets of the form $\mathcal{P}(\pi)$, where $\pi$ is a nonzero period.

From Corollary 6.7 we easily see that for each $n \in \mathbb{N}$ the point

$$
\mathbf{Q}_{n}:=\left(0, \ldots, 0,1-\frac{1}{2 n^{2}},-\frac{2 n+1}{2 n(n+1)}\right) \in \mathbb{R}^{d}
$$

is not contained in $\mathcal{D}_{d}^{0}$. Moreover, since $\mathbf{Q}_{n} \rightarrow \mathbf{K}_{d}$ for $n \rightarrow \infty$ there exists some $n \in \mathbb{N}$ such that $\mathbf{Q}_{n} \in U$ for all $n \geq N$. Summing up we have

$$
\mathbf{Q}_{n} \in U \backslash \mathcal{D}_{d}^{0} \quad \text { for all } \quad n \geq N \text {. }
$$

Suppose that we can cover $U \backslash \mathcal{D}_{d}^{0}$ by finitely many cutout polyhedra. Let $\pi_{1}, \ldots, \pi_{M}$ be the corresponding periods. Then

$$
\pi_{i}:\left(a_{i, 1}, \ldots, a_{i, d}\right) ; a_{i, d+1}, \ldots, a_{i, L_{i}} \quad(1 \leq i \leq M) .
$$

Let

$$
K>\max _{1 \leq i \leq M} \max _{1 \leq j \leq L_{i}}\left|a_{i, j}\right| \text {. }
$$

Since the sequences $\left\{1-\frac{1}{2 n^{2}}\right\}_{n \geq 1}$ and $\left\{-\frac{2 n+1}{2 n(n+1)}\right\}_{n \geq 1}$ meet the conditions of Lemma 7.4 we conclude that there exists some $N^{\prime} \in \mathbb{N}$ such that

$$
\mathbf{Q}_{n} \notin \bigcup_{1 \leq i \leq M} \mathcal{P}\left(\pi_{i}\right) \quad \text { for all } n \geq N^{\prime} .
$$

If we take $n \geq \max \left(N, N^{\prime}\right)$, relations (7.4) and (7.5) can not hold simultaneously, which gives the desired contradiction. 
Remark 7.6. By similar reasoning one can also show that for each $d \geq 2$ the point

$$
\mathbf{K}_{d}^{\prime}:=(0, \ldots, 0,1,1)
$$

is a critical point of $\mathcal{D}_{d}$.

This yields that the sets $\mathcal{D}_{d}^{0}$ have a complicated structure near $\mathbf{K}_{d}$ and $\mathbf{K}_{d}^{\prime}$.

Corollary 7.7. For $d \geq 2$ the family of cutout polyhedra which has to be cut out from $\mathcal{D}_{d}$ in order to obtain $\mathcal{D}_{d}^{0}$ must be infinite.

For $d \geq 3$ the family of cutout polyhedra which has to be cut out from $\mathcal{C}_{d}$ in order to obtain $\mathcal{C}_{d}^{0}$ must be infinite.

Summing up we can say that characterizing the set $\mathcal{D}_{d}^{0}$ can be done by cutting out finitely many polyhedra everywhere apart from neighborhoods of critical points. Thus the solution of the following problem would be a big step towards a characterization of $\mathcal{D}_{d}^{0}$.

Problem 7.8. Characterize the critical points of $\mathcal{D}_{d}$. Can one show that for a given $d$ there exist only finitely many critical points?

SRS seem to be a good foundation for a unified treatment of deep problems in numeration systems. Based on the general results presented in this note we are therefore aiming at more explicit results for dimension 2 in a forthcoming paper.

\section{REFERENCES}

[1] S. AкiYama, Self affine tiling and Pisot numeration system, "Number Theory and its Applications", ed. by K. Győry and S. Kanemitsu, Kluwer (1999), 7-17.

[2] S. AkiYama, Cubic Pisot units with finite beta expansions, "Algebraic Number Theory and Diophantine Analysis", ed. F. Halter-Koch and R. F. Tichy, de Gruyter (2000), 11-26.

[3] S. AkiYama, On the boundary of self affine tilings generated by Pisot numbers, Journal of Math. Soc. Japan, 54, no. 2 (2002), 283-308.

[4] S. Akiyama, H. Brunotte and A. Ретнö, Cubic CNS polynomials, notes on a conjecture of W. J. Gilbert, J. Math. Anal. and Appl., 281 (2003), 402-415.

[5] S. Akiyama and N. GJini, Connectedness of number theoretic tilings, preprint.

[6] S. Akiyama and A. Pethö, On canonical number systems, Theoret. Comput. Sci., 270 (2002), 921 - 933.

[7] S. Akiyama and H. Rao, New criteria for canonical number systems, Acta Arith., 111, no. 1 (2004), 5-25.

[8] S. Akiyama, H. Rao and W. Steiner, A certain finiteness property of Pisot number system, J. Number Theory, 107 (2004), no. 1, 135-160.

[9] A. Bertrand, Développement en base de Pisot et répartition modulo 1, C. R. Acad. Sci. Paris Sér. A-B, 285 (1977), 419-421.

[10] T. BorbéLy, Általánosított számrendszerek, Master Thesis, University of Debrecen, 2003.

[11] H. Brunotte, On trinomial bases of radix representations of algebraic integers, Acta Sci. Math. (Szeged), 67 (2001), 521-527.

[12] C. Frougny and B. Solomyak, Finite beta-expansions, Ergod. Th. and Dynam. Sys., 12 (1992), 713-723.

[13] W. J. Gilbert, Radix representations of quadratic fields, J. Math. Anal. Appl., 83 (1981), 264-274.

[14] V. GrünWALD, Intorno all'aritmetica dei sistemi numerici a base negativa con particolare riguardo al sistema numerico a base negativo-decimale per lo studio delle sue analogie coll'aritmetica ordinaria (decimale), Giornale di matematiche di Battaglini, 23 (1885), 203-221, 367.

[15] M. Hollander, Linear Numeration Systems, Finite Beta Expansions, and Discrete Spectrum of Substitution Dynamical Systems, PhD Thesis, Washington University.

[16] I. KÁtAi AND B. KovÁCS, Kanonische Zahlensysteme in der Theorie der quadratischen algebraischen Zahlen, Acta Sci. Math. (Szeged), 42 (1980), 99-107.

[17] I. Kátai And B. Kovács, Canonical number systems in imaginary quadratic fields, Acta Math. Acad. Sci. Hungar., 37 (1981), 159-164.

[18] I. KÁtai And J. Szabó, Canonical number systems for complex integers, Acta Sci. Math. (Szeged), 37 (1975), $255-260$.

[19] I. KÁtAI, Number systems in imaginary quadratic fields, Festschrift for the 50th birthday of Karl-Heinz Indlekofer. Ann. Univ. Sci. Budapest. Sect. Comput., 14 (1994), 91-103.

[20] D. E. KNUth, An imaginary number system, Comm. ACM, 3 (1960), 245-247.

[21] D. E. Knuth, The Art of Computer Programming, Vol. 2 Semi-numerical Algorithms, Addison Wesley (1998) London 3rd-edition.

[22] A. KovÁcs, Generalized binary number systems, Ann. Univ. Sci. Budap. Rolando Etvös, Sect. Comput., 20 (2001), 195-206.

[23] B. Kovács, CNS rings, Colloq. Math. Soc. János Bolyai, 34 (1981), 961-971. 
[24] B. KovÁcs, Canonical number systems in algebraic number fields, Acta Math. Acad. Sci. Hungar., 37 (1981), 405-407.

[25] B. Kovács And A. Pethő, Canonical systems in the ring of integers, Publ. Math. Debrecen, 30 (1983), 39-45.

[26] B. Kovács AND A. Ретнő, Number systems in integral domains, especially in orders of algebraic number fields, Acta Sci. Math. (Szeged), 55 (1991), 287-299.

[27] J. Lagarias and Y.Wang, Self affine Tiles in $\mathbb{R}^{n}$, Adv. Math., 121, no. 1(1996), 21-49.

[28] G. MichaleK, Base $N$ just touching covering systems, Publ. Math. Debrecen, 58 (2001), 549-557.

[29] W. Parry, On the $\beta$-expansions of real numbers, Acta Math. Acad. Sci. Hungar., 11 (1960), 401-416.

[30] W. Penney, A "binary" system for complex numbers, J. Assoc. Comput. Mach., 12 (1965), 247-248.

[31] A. Ретнӧ, On a polynomial transformation and its application to the construction of a public key cryptosystem, Computational Number Theory, Proc., Eds.: A. Pethő, M. Pohst, H. G. Zimmer and H. C. Williams, Walter de Gruyter Publ. Comp. (1991), 31-43.

[32] A. Pетнӧ, Notes on CNS polynomials and integral interpolation, to appear.

[33] B. Praggastis, Markov partition for hyperbolic toral automorphism, PhD Thesis, Washington University, 1992.

[34] A. RÉNYI, Representations for real numbers and their ergodic properties, Acta Math. Acad. Sci. Hungar., 8 (1957), 477-493.

[35] K. SCHeICHER, Kanonische Ziffernsysteme und Automaten, Grazer Math. Ber., 333 (1997), 1-17.

[36] K. Scheicher And J. M. Thuswaldner, On the characterization of canonical number systems, Osaka J. Math., 41, no. 2 (2004), 327-351.

[37] K. Scheicher And J. M. Thuswaldner, Digit systems in polynomial rings over finite fields, Finite Fields Appl., 9 (2003), 322-333.

[38] K. Sснмidt, On periodic expansions of Pisot numbers and Salem numbers, Bull. London Math. Soc., 12 (1980), 269-278.

[39] I. SchUR, Über Potenzreihen, die im Inneren des Einheitskreises beschränkt sind II, J. reine angew. Math., 148 (1918), $122-145^{2}$

[40] T. TAKagI, Lectures in Algebra, 1965 (in Japanese).

[41] W. P. Thurston, Groups, Tilings and Finite state automata, AMS Colloquium lectures, 1989.

[42] J. M. Thuswaldner, Elementary properties of canonical number systems in quadratic fields, Applications of Fibonacci Numbers, Volume 7, G. E. Bergum et al. (eds.), Kluwer Academic Publishers (1998), 405-414.

Department of Mathematics, Faculty of Science Niigata University, Ikarashi 2-8050, Niigata 9502181, JAPAN

E-mail address: akiyama@math.sc.niigata-u.ac.jp

National Instruments Europe Kft, H-4031 Debrecen Hatar u. 1/A, HUnGary

E-mail address: tibor.borbely@ni.com

Haus-Endt-Strasse 88, D-40593 Düsseldorf, GERMAnY

E-mail address: brunoth@web.de

Department of Computer Science, University of Debrecen, P.O. Box 12, H-4010 Debrecen, Hungary E-mail address: pethoe@inf.unideb.hu

Department of Mathematics and Statistics, Leoben University, Franz-Josef-Strasse 18, A-8700 Leoben, AUSTRIA

E-mail address: joerg.thuswaldner@unileoben.ac.at

\footnotetext{
${ }^{2}$ Reprinted in Schur's collected papers.
} 